\title{
DOCUMENTATION AND CONSERVATION OF ETHNOMEDICINAL PRACTICES IN TRIBAL PEOPLE OF CHITRAKOOT REGION
}

\author{
RASHMI SINGH $^{1^{*}}$ and MANOJ TRIPATHI ${ }^{2}$
}

${ }^{1}$ Department of Botany, Govt. Autonomous P. G. College, Satna (M.P.)

${ }^{2}$ Deendayal Research Institute, Arogyadham, Chitrakoot (M.P.)

\begin{abstract}
A study on ethnomedicinal practices used by tribal people was conducted from April,2018-March, 2019 in Chitrakoot district of M.P. The study was focused on the treatment of various diseases through medicinal plants found in the area, used by tribal people. We documented the plant parts used for drug formulations, method of preparation of drug, method of administration of drug and also the dose and duration of drug uses. The data was collected from 18 tribal dominant villages, 674 interviews were conducted through 86 intensive field visits, covering almost all seasons of the year.
\end{abstract}

Key words : Ethnomedicinal practices, Tribal people, Treatment of diseases

\section{INTRODUCTION}

India is a repository of medicinal plants due to varied climatic conditions. India is rich in its floristic wealth. Tribal people have been using plants as medicines since time immortal. Traditional herbal medicines play a significant role in the treatment of various common ailments and diseases. The present study is based on the conservation of vanishing traditional knowledge of ethnomedicinal practices done by tribals of Chitrakoot region. Jain (1991) given an enormous information on medicinal plants in his book "Dictionary of Indian folk medicines and ethnobotany". Chopra et al. (1956) also given a Glossary of Indian medicinal plants.

There are two streams of traditional knowledge of ethnomedicinal plants are prevalent in India. One is well documented and other is in the form of folklore or undocumented. Examples of first one are Ayurveda, Unnani, Homeopathy and of second one are transmitted orally from one generation to other, which is very common among tribal people. This stream still play a significant role in the treatment of various ailment and diseases, particularly in tribal and rural area of India.

\section{MATERIAL AND METHODS}

The study site is Majhgawan Tehsil of Chitrakoot, Satna district, M.P. It lies between $80^{\circ} 52^{\prime \prime}-80^{\circ} 73^{\prime \prime N}$ latitude and $25^{\circ} 10^{\prime \prime}-25^{\circ} 52^{\prime \prime E}$ longitude. The majority of population belong to Kol, Gond, Khairwar and Mawasi tribes. They generally reside in and around the forest areas. Around $79 \%$ of total population of Majhgawan Tehsil lives in rural areas. Schedule Caste (SC) constitutes $15 \%$ while Schedule Tribe (ST) is $28.8 \%$ of total population in Majhgawan Tehsil. This region has tropical mixed dry deciduous type of forest.

For documentation of folklore/traditional medicinal knowledge in Chitrakoot region 18 tribal dominant villages were selected for survey, these are Amiliya, Barua,
Bhargawan, Bundelapur, Chandai, Devlaha, Tagi, Hiraundi, Kailashpur, Kathauta, Koldari, Parewa, Patna, Patni, Pindra, Piparitola, Raiya and Turra. 674 interviews were conducted through 86 intensive field visits during April,2018March,2019, covering almost all the seasons of the year.

The data was also collected through exploration of deep forest with Traditional Medicine Practitioner (TMP) to find out exact location of medicinal plants. Digital photography has been done for reference and further studies (Plate.1).

The detailed information such as plant name, parts used, mode of preparation, mode of application, dose and duration etc. has been collected with the help of prescribed proforma. The voucher specimen were also collected and identified with the help of local flora; and preserved in the herbarium of Arogyadham, Deendayal Research Institute, Chitrakoot. The recorded medicinal uses of various plants were compared with the well known Indian literature and found that these uses are not recorded in the consulted literature.

For identification of these medicinal plants various flora's were used, such as flora of Madhya Pradesh by Verma et al.,1993; Singh et al.,2001 and Mudgal et al.,1997. Dictionary of Indian folk medicines and ethnobotany by Jain and Rao,1977 was a milestone for this study. For identification of local plants Sikarwar (2001) and Sikarwar et al. (2008) gives an immense view on ethnobotany of Madhya Pradesh.

\section{RESULTS AND DISCUSSION}

It was observed that instead of single drug used by the local and tribal people of Chitrakoot, the Vaidhyas/traditional healers, generally use combined formulations of medicinal plants (Table.1). Because they believe that the compound formulations have synergistic effects. Total 252 formulations are being used for the treatment of 51 various diseases viz. Bone fracture \& Sprain (15 formulations), Measles \& chickenpox 
Table. 1 Folklore/traditional practices used by tribal's of Chitrakoot region for treatment for various diseases.

Note : These formulations were obtained through survey in the study sites. These are general formulations, but it is an appeal that don't take these formulations without prescription of authorised medical practitioners obtained after thorough check up because these medicines may vary from one person to an other. It may suits to one persons, but may not suit to other.

\begin{tabular}{|c|c|c|c|c|c|c|}
\hline S. & $\begin{array}{l}\text { Name of } \\
\text { Diseases }\end{array}$ & $\begin{array}{l}\text { Ingredients/ } \\
\text { formulations name }\end{array}$ & Botanical name and family & $\begin{array}{l}\text { Method of } \\
\text { preparation }\end{array}$ & $\begin{array}{l}\text { Method of } \\
\text { administration }\end{array}$ & $\begin{array}{l}\text { Dose \& } \\
\text { duration }\end{array}$ \\
\hline \multirow[t]{13}{*}{1.} & \multirow[t]{13}{*}{$\begin{array}{l}\text { Bone } \\
\text { Fracture } \\
\text { and } \\
\text { Sprain }\end{array}$} & $\begin{array}{l}\text { Haldi (rhizome); } \\
\text { Chuna (Calcium carbonate) } \\
\text { Haldi (rhizome), } \\
\text { Chuna (Calcium carbonate) } \\
\text { Honey }\end{array}$ & $\begin{array}{l}\text { Curcuma longa Linn. } \\
\text { (Zingiberaceae) } \\
\text { Curcuma longa Linn. } \\
\text { (Zingiberaceae) }\end{array}$ & $\begin{array}{l}\text { Mix the ingredients } \\
\& \text { make a paste } \\
\text { Mix the ingredients } \\
\& \text { make a paste }\end{array}$ & $\begin{array}{l}\text { Apply to the } \\
\text { affected area } \\
\text { Apply to the } \\
\text { affected area }\end{array}$ & $\begin{array}{l}\text { Twice a day } \\
\text { for 5-7 days } \\
\text { Twice a day } \\
\text { for 3-5 days }\end{array}$ \\
\hline & & $\begin{array}{l}\text { Haldi (rhizome), } \\
\text { Ajwain (fruit), } \\
\text { Onion (rhizome) }\end{array}$ & $\begin{array}{l}\text { C.longa Linn. (Zingiberaceae); } \\
\text { T.ammi (L.) (Apiaceae); } \\
\text { Allium cepa Linn.(Liliaceae) }\end{array}$ & $\begin{array}{l}\text { Mix the ingredients } \\
\& \text { make a paste }\end{array}$ & $\begin{array}{l}\text { Apply to the } \\
\text { affected area }\end{array}$ & $\begin{array}{l}\text { Twice a day } \\
\text { for } 3-5 \text { days }\end{array}$ \\
\hline & & $\begin{array}{l}\text { Mahuwa (flower), } \\
\text { Madar (leaves) }\end{array}$ & $\begin{array}{l}\text { M.longifolia (Koenig) Macb. } \\
\text { (Sapotaceae); C.procera (Aiton.) } \\
\text { Dryand. (Asclepiadaceae) }\end{array}$ & $\begin{array}{l}\text { Grind the ingredients } \\
\& \text { make a paste }\end{array}$ & $\begin{array}{l}\text { Apply to the } \\
\text { affected area }\end{array}$ & $\begin{array}{l}\text { Twice a day } \\
\text { for } 3-5 \text { days }\end{array}$ \\
\hline & & Ghamira (leaves), & $\begin{array}{l}\text { Eclipta prostrata }(\mathrm{L} .) \\
\text { (Asteraceae) }\end{array}$ & Extract the juice & $\begin{array}{l}\text { Apply to the } \\
\text { affected area }\end{array}$ & $\begin{array}{l}\text { Twice a day } \\
\text { for } 3-5 \text { days }\end{array}$ \\
\hline & & $\begin{array}{l}\text { Amahaldi (rhizome); } \\
\text { Ajwain (fruit), } \\
\text { Menda (stem bark) }\end{array}$ & $\begin{array}{l}\text { C.amada Roxb. (Zingiberaceae); } \\
\text { T.ammi (L.) (Apiaceae); } \\
\text { L.glutinosa (Lour) Robins. } \\
\text { (Lauraceae) }\end{array}$ & $\begin{array}{l}\text { Grind the ingredients } \\
\& \text { make a paste }\end{array}$ & $\begin{array}{l}\text { Apply to the } \\
\text { affected area }\end{array}$ & $\begin{array}{l}\text { Twice a day } \\
\text { for } 3-5 \text { days }\end{array}$ \\
\hline & & $\begin{array}{l}\text { Haldi (rhizome), } \\
\text { Chuna (Calcium carbonate) } \\
\text { Hadjod (aerial part) }\end{array}$ & $\begin{array}{l}\text { C.longa Linn. (Zingiberaceae); } \\
\text { C.quandrangularis L. (Vitaceae) }\end{array}$ & $\begin{array}{l}\text { Grind the ingredients } \\
\& \text { make a paste }\end{array}$ & $\begin{array}{l}\text { Apply to the } \\
\text { affected area }\end{array}$ & $\begin{array}{l}\text { Twice a day } \\
\text { for } 3-5 \text { days }\end{array}$ \\
\hline & & $\begin{array}{l}\text { Mahua (leaves), } \\
\text { Hadjod (aerial part) }\end{array}$ & $\begin{array}{l}\text { Madhuca longifolia (Koenig) } \\
\text { Macb. (Sapotaceae); } \\
\text { C.quandragularis L. (Vitaceae) }\end{array}$ & $\begin{array}{l}\text { Grind the ingredients } \\
\& \text { make a paste }\end{array}$ & $\begin{array}{l}\text { Apply to the } \\
\text { affected area }\end{array}$ & $\begin{array}{l}\text { Twice a day } \\
\text { for } 5 \text { days }\end{array}$ \\
\hline & & $\begin{array}{l}\text { Amahaldi (rhizome) } \\
\text { Mehandi (root) } \\
\text { Mahua (flower), } \\
\text { Haldi (rhizome), } \\
\text { Ajawain (fruit), } \\
\text { Gud }\end{array}$ & $\begin{array}{l}\text { C.amada Roxb. (Zingiberaceae); } \\
\text { L.inermis L. (Lythraceae) } \\
\text { M.longifolia (Koenig) } \\
\text { Macb. (Sapotaceae); } \\
\text { C.longa Linn. (Zingiberaceae); } \\
\text { T.ammi (L.) (Apiaceae); }\end{array}$ & $\begin{array}{l}\text { Grind the ingredients } \\
\& \text { make a paste } \\
\text { Grind the ingredients } \\
\& \text { make a paste }\end{array}$ & $\begin{array}{l}\text { Apply in the } \\
\text { affected area } \\
\text { Apply in the } \\
\text { affected area }\end{array}$ & $\begin{array}{l}\text { Twice a day } \\
\text { for } 2-5 \text { days } \\
\text { Twice a day } \\
\text { for } 2-5 \text { days }\end{array}$ \\
\hline & & Tendu (stem bark) & $\begin{array}{l}\text { Diospyros esculpta (Gaertn) } \\
\text { (Ebenaceae) }\end{array}$ & $\begin{array}{l}\text { Grind it and } \\
\text { make a paste }\end{array}$ & $\begin{array}{l}\text { Apply in the } \\
\text { affected area }\end{array}$ & $\begin{array}{l}\text { Twice a day } \\
\text { for } 1-3 \text { days }\end{array}$ \\
\hline & & $\begin{array}{l}\text { Ajowan (fruit), } \\
\text { Haldi (rhizome), } \\
\text { Madar (leaves), } \\
\text { Dhatura (leaves), } \\
\text { Mahua (flower) }\end{array}$ & $\begin{array}{l}\text { T.ammi (L.) (Apiaceae); } \\
\text { C.longa Linn. (Zingiberaceae); } \\
\text { C.procera (Aiton.) Dryand. } \\
\text { (Asclepiadaceae); Datura metel } \\
\text { L. (Solanaceae); M.longifolia } \\
\text { (Koenig) Macb. (Sapotaceae); }\end{array}$ & $\begin{array}{l}\text { Roast all the } \\
\text { ingredients and } \\
\text { make a pultish }\end{array}$ & $\begin{array}{l}\text { Use the pultish } \\
\text { for Sudation of } \\
\text { the affected area }\end{array}$ & $\begin{array}{l}\text { Twice a day } \\
\text { for } 2-5 \text { days }\end{array}$ \\
\hline & & Babool (fruit) & $\begin{array}{l}\text { Acacia nilotica } \\
\text { (Mimosaceae) }\end{array}$ & $\begin{array}{l}\text { Grind the fruits to } \\
\text { make a powder }\end{array}$ & $\begin{array}{l}\text { Take the powder } \\
\text { with water }\end{array}$ & $\begin{array}{l}1-2 \text { gm twice a } \\
\text { day for 5-7 days }\end{array}$ \\
\hline & & $\begin{array}{l}\text { Meda (bark), } \\
\text { Gurubel (Leaf) }\end{array}$ & $\begin{array}{l}\text { Litsea glutinosa (Lauraceae); } \\
\text { T.cordifolia (Menispermaceae) }\end{array}$ & $\begin{array}{l}\text { Grind it and } \\
\text { make a paste }\end{array}$ & $\begin{array}{l}\text { Apply on the } \\
\text { affected area }\end{array}$ & $\begin{array}{l}\text { Twice a day } \\
\text { for } 1-3 \text { days }\end{array}$ \\
\hline & & Baghnakhi (leaves) & $\begin{array}{l}\text { Martynia annua } \\
\text { (Martyniaceae) }\end{array}$ & $\begin{array}{l}\text { Grind it and } \\
\text { a paste }\end{array}$ & $\begin{array}{l}\text { Apply on the } \\
\text { affected area }\end{array}$ & $\begin{array}{l}\text { Twice a day } \\
\text { for } 1-3 \text { days }\end{array}$ \\
\hline \multirow[t]{4}{*}{2.} & \multirow{4}{*}{$\begin{array}{l}\text { Measles } \\
\text { and } \\
\text { Chiken } \\
\text { pox }\end{array}$} & $\begin{array}{l}\text { Haldi (rhizome), } \\
\text { Durba (aerial part), }\end{array}$ & $\begin{array}{l}\text { C.longa Linn. (Zingiberaceae); } \\
\text { C.dactylon (L.) Pers (Poaceae); }\end{array}$ & $\begin{array}{l}\text { Grind, mix with oil } \\
\text { to make a paste }\end{array}$ & $\begin{array}{l}\text { Apply on the } \\
\text { affected area }\end{array}$ & $\begin{array}{l}\text { Twice a day } \\
\text { for } 1-3 \text { days }\end{array}$ \\
\hline & & $\begin{array}{l}\text { Haldi (rhizome), } \\
\text { Mustard oil }\end{array}$ & $\begin{array}{l}\text { C.longa Linn. (Zingiberaceae); } \\
\text { B.compestris Linn.var. sarson } \\
\text { Prain (Brassicaceae) }\end{array}$ & Grind, mix with oil & Apply on the & Twice a day \\
\hline & & $\begin{array}{l}\text { Duba, } \\
\text { Swate chandan }\end{array}$ & $\begin{array}{l}\text { C.dactylon (L.) Pers (Poaceae); } \\
\text { S.album Linn. (Santalaceae) }\end{array}$ & $\begin{array}{l}\text { Grind to make } \\
\text { a paste }\end{array}$ & $\begin{array}{l}\text { Apply on the } \\
\text { affected area }\end{array}$ & $\begin{array}{l}\text { Twice a day } \\
\text { for } 1-3 \text { days }\end{array}$ \\
\hline & & Amla (leaf) & $\begin{array}{l}\text { Phyllanthus emblica } \\
\text { (Phyllanthaceae) }\end{array}$ & $\begin{array}{l}\text { Grind to make } \\
\text { a extract }\end{array}$ & $\begin{array}{l}\text { Take the } \\
\text { extract }\end{array}$ & $\begin{array}{l}\text { Twice a day } \\
\text { for } 1-3 \text { days }\end{array}$ \\
\hline \multirow[t]{3}{*}{3.} & \multirow[t]{3}{*}{ Jaundice } & $\begin{array}{l}\text { Papaya (fruit), } \\
\text { Spinach (leaves), } \\
\text { Radish (root), } \\
\text { Carrot (root), } \\
\text { Gram (leaf) }\end{array}$ & $\begin{array}{l}\text { H.laurifolia (Dennst.) (Flacourtiaceae); } \\
\text { Spinach oleracea (Amaranthaceae); } \\
\text { Radish sativus (Brassicaceae); } \\
\text { Daucus carota (Apiaceae); } \\
\text { Cicer arietinum } \\
\text { (Fabaceae) }\end{array}$ & $\begin{array}{l}\text { Wash all the } \\
\text { ingredients }\end{array}$ & Directly eat it & $250 \mathrm{gm}$ \\
\hline & & $\begin{array}{l}\text { Mango (leaves), } \\
\text { Jamun (leaves) \& } \\
\text { Arjun (stem bark) }\end{array}$ & $\begin{array}{l}\text { M.indica (Anacardiaceae); } \\
\text { S.cumini (Linn) Skeels } \\
\text { (Myrtaceae); Terminelia arjuna } \\
\text { (Combreta) (Combretaceae) }\end{array}$ & $\begin{array}{l}\text { Boil with water and } \\
\text { take a bath, }\end{array}$ & $\begin{array}{l}\text { Directly take } \\
\text { a bath }\end{array}$ & $\begin{array}{l}\text { Once a day } \\
\text { for 5-7 days }\end{array}$ \\
\hline & & $\begin{array}{l}\text { Pumpkin (fruit), } \\
\text { Spinach (leaves), } \\
\text { Radish (root), }\end{array}$ & $\begin{array}{l}\text { L.siceraria }(\mathrm{Mol}) \text { (Cucurbitaceae) } \\
\text { S.oleracea (Chenopodiaceae); } \\
\text { R.sativus (Brassicaceae); }\end{array}$ & $\begin{array}{l}\text { Wash all the } \\
\text { ingredients }\end{array}$ & $\begin{array}{l}\text { Drink the juice } \\
\text { and directly }\end{array}$ & $\begin{array}{l}250 \mathrm{ml} \text { juice } \\
\text { twice a day for } \\
5-7 \text { days }\end{array}$ \\
\hline
\end{tabular}




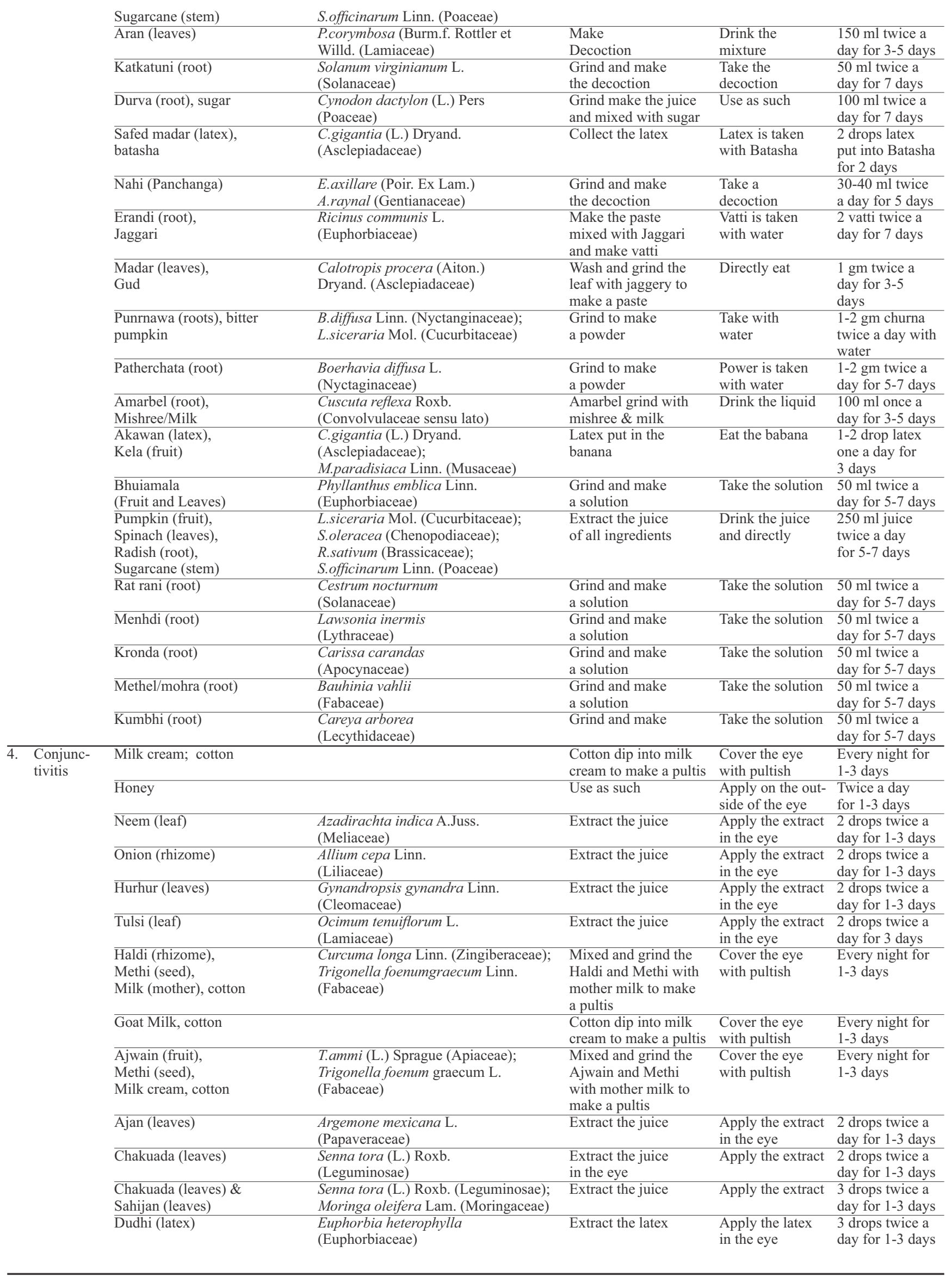




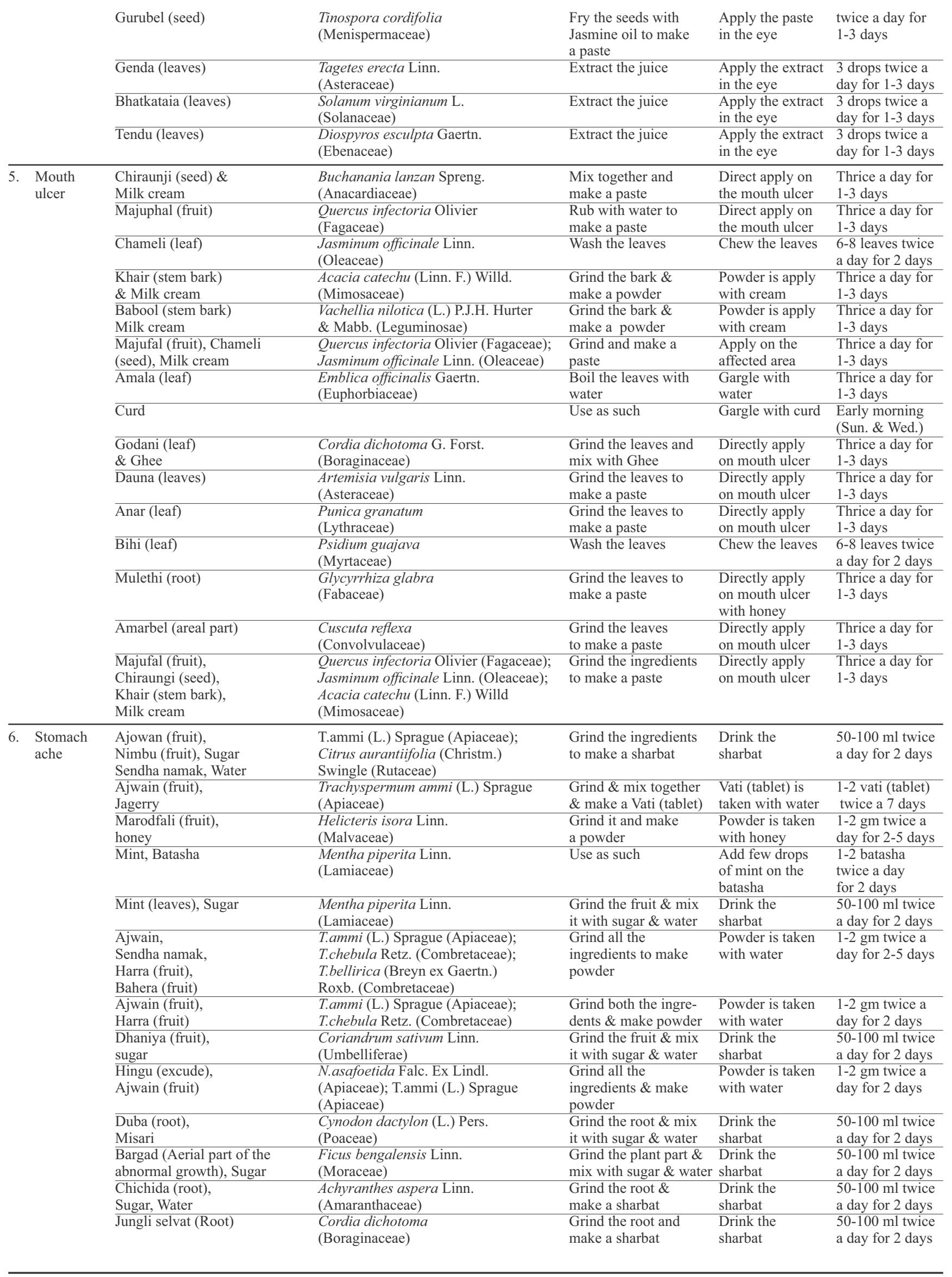




\begin{tabular}{|c|c|c|c|c|c|c|}
\hline & & $\begin{array}{l}\text { Tinsa (root), } \\
\text { Mahua (root) }\end{array}$ & $\begin{array}{l}\text { Ougeinia oojeinensis (Fabaceae); } \\
\text { Mahua longifolia (Sapotaceae) }\end{array}$ & $\begin{array}{l}\text { Grind the root to } \\
\text { make a powder }\end{array}$ & $\begin{array}{l}\text { Take the } \\
\text { powder }\end{array}$ & $\begin{array}{l}1-2 \text { gm powder } \\
\text { twice a day for } \\
1-5 \text { days }\end{array}$ \\
\hline & & $\begin{array}{l}\text { Padin (root), } \\
\text { Kaduwa kanda (root) }\end{array}$ & $\begin{array}{l}\text { Cissampelos pareira (Menispermaceae); } \\
\text { Dioscorea bulbifera (Dioscoreaceae) }\end{array}$ & $\begin{array}{l}\text { Grind the root to } \\
\text { make a powder }\end{array}$ & $\begin{array}{l}\text { Take the } \\
\text { powder }\end{array}$ & $\begin{array}{l}1-2 \text { gm powder } \\
\text { twice a day for } \\
1-5 \text { days }\end{array}$ \\
\hline & & Bendar (root) & $\begin{array}{l}\text { Gardenia gummifera } \\
\text { (Rubiaceae) }\end{array}$ & Make the decoction & $\begin{array}{l}\text { Take the } \\
\text { decoction }\end{array}$ & $\begin{array}{l}40-50 \mathrm{ml} \text { twice } \mathrm{a} \\
\text { day for } 1-3 \text { days }\end{array}$ \\
\hline 7. & Ear pain & Hurhur (leaf) & $\begin{array}{l}\text { Gynandropsis gynandra (Linn.) } \\
\text { (Cleomaceae) }\end{array}$ & Extract the juice & $\begin{array}{l}\text { Apply the extract } \\
\text { in the ear }\end{array}$ & $\begin{array}{l}10 \text { drops twice a } \\
\text { day for } 1-3 \text { days }\end{array}$ \\
\hline & & Genda (leaf) & $\begin{array}{l}\text { Tagetes erecta Linn. } \\
\text { (Asteraceae) }\end{array}$ & Extract the juice & $\begin{array}{l}\text { Apply the extract } \\
\text { in the ear }\end{array}$ & $\begin{array}{l}10 \text { drops twice a } \\
\text { day for } 1-3 \text { days }\end{array}$ \\
\hline & & $\begin{array}{l}\text { Lahsun (bulb), } \\
\text { Hingu (exude), } \\
\text { Mustard oil }\end{array}$ & $\begin{array}{l}\text { Allium sativum Linn. (Amaryllidaceae); } \\
\text { Narthex asafoetida Falc. Ex Lind. } \\
\text { (Apiaceae); B.campestris Linn. Var. } \\
\text { sarson Prain (Brassicaceae) }\end{array}$ & $\begin{array}{l}\text { Lasun and Hingu } \\
\text { gently boiled in } \\
\text { mustard oil }\end{array}$ & $\begin{array}{l}\text { Lukewarm oil } \\
\text { apply in the ear }\end{array}$ & $\begin{array}{l}10 \text { drops thrice a } \\
\text { day for } 1-3 \text { days }\end{array}$ \\
\hline & & Bakain (leaves) & $\begin{array}{l}\text { Melia azedarach L. } \\
\text { (Meliaceae) }\end{array}$ & $\begin{array}{l}\text { Grind the leaf to } \\
\text { make a juice }\end{array}$ & $\begin{array}{l}\text { Apply the extract } \\
\text { in the ear }\end{array}$ & $\begin{array}{l}4-5 \text { drops, apply } \\
\text { in to ear twice a } \\
\text { day for } 2 \text { days }\end{array}$ \\
\hline & & Tulsi (leaf) & $\begin{array}{l}\text { Ocimum tenuiflorum } \mathrm{L} . \\
\text { (Lamiaceae) }\end{array}$ & Extract the juice & $\begin{array}{l}\text { Apply the extract } \\
\text { in the ear }\end{array}$ & $\begin{array}{l}4-5 \text { drops twice a } \\
\text { day for } 1-3 \text { days }\end{array}$ \\
\hline & & Sudarshan (leaf) & $\begin{array}{l}\text { Crinum latifolium Linn. } \\
\text { (Amaryllidaceae) }\end{array}$ & Extract the juice & $\begin{array}{l}\text { Apply the extract } \\
\text { in the ear }\end{array}$ & $\begin{array}{l}\text { 4-5 drops twice a } \\
\text { day for } 1-3 \text { days }\end{array}$ \\
\hline & & $\begin{array}{l}\text { Sehund (leaf), } \\
\text { Hingu (exude) }\end{array}$ & $\begin{array}{l}\text { E.nerrifolia Linn. (Euphorbiaceae); } \\
\text { N.asafetida Falc. Ex Lindl. (Apiaceae) }\end{array}$ & $\begin{array}{l}\text { Extract the juice } \\
\text { and gently heat }\end{array}$ & $\begin{array}{l}\text { Lukewarm extract } \\
\text { apply in the ear }\end{array}$ & $\begin{array}{l}10 \text { drop thrice a } \\
\text { day for } 1-3 \text { days }\end{array}$ \\
\hline & & Chichida (leaf) & $\begin{array}{l}\text { Achyranthes aspera Linn. } \\
\text { (Amaranthaceae) }\end{array}$ & Extract the juice & $\begin{array}{l}\text { Apply the extract } \\
\text { in the ear }\end{array}$ & $\begin{array}{l}5-6 \text { drops twice a } \\
\text { day for } 1-3 \text { days }\end{array}$ \\
\hline & & Amarbel (areal part) & $\begin{array}{l}\text { Cuscuta reflexa } \\
\text { (Convolvulaceae) }\end{array}$ & Extract the juice & $\begin{array}{l}\text { Apply the extract } \\
\text { in the ear }\end{array}$ & $\begin{array}{l}\text { 5-6 drops twice a } \\
\text { day for } 1-3 \text { days }\end{array}$ \\
\hline & & Onion (bulb) & $\begin{array}{l}\text { Allium cepa } \\
\text { (Amaryllidaceae) }\end{array}$ & Extract the juice & $\begin{array}{l}\text { Apply the extract } \\
\text { in the ear }\end{array}$ & $\begin{array}{l}5-6 \text { drops twice a } \\
\text { day for } 1-3 \text { days }\end{array}$ \\
\hline & & Kapash (leaf) & $\begin{array}{l}\text { Gossypium herbaceum Linn. } \\
\text { (Malvaceae) }\end{array}$ & Extract the juice & $\begin{array}{l}\text { Apply the extract } \\
\text { in the ear }\end{array}$ & $\begin{array}{l}5-6 \text { drops twice a } \\
\text { day for } 1-3 \text { days }\end{array}$ \\
\hline 8. & $\begin{array}{l}\text { Skin } \\
\text { disease }\end{array}$ & $\begin{array}{l}\text { Kalimirch (seed), } \\
\text { Neem (stem bark), } \\
\text { Mustard oil }\end{array}$ & $\begin{array}{l}\text { P.nigrum L. (Piperaceae); A.indica } \\
\text { A.Juss. (Meliaceae); B.campestris } \\
\text { L. var. sarson Prain (Brassicaceae) }\end{array}$ & $\begin{array}{l}\text { Grind and gently } \\
\text { boiled with mustard } \\
\text { oil }\end{array}$ & $\begin{array}{l}\text { Lukewarm oil } \\
\text { apply in the } \\
\text { affected area }\end{array}$ & $\begin{array}{l}\text { Twice a day for } \\
\text { for } 4-5 \text { days }\end{array}$ \\
\hline & & Chilla (stem bark) & $\begin{array}{l}\text { Holoptelea integrifolia (Roxb.) } \\
\text { Planch. (Ulmaceae) }\end{array}$ & $\begin{array}{l}\text { Grind the bark to } \\
\text { make a paste }\end{array}$ & $\begin{array}{l}\text { Apply in the } \\
\text { affected area }\end{array}$ & $\begin{array}{l}\text { Twice a day for } \\
3-5 \text { days }\end{array}$ \\
\hline & & $\begin{array}{l}\text { Mustard (oil), } \\
\text { Nimbu (fruit) }\end{array}$ & $\begin{array}{l}\text { B.compestris L. var. sarson Prain } \\
\text { (Brassicaceae); C.aurantiifolia } \\
\text { (Christm.) Swingle (Rutaceae) }\end{array}$ & $\begin{array}{l}\text { Mixed both } \\
\text { ingredients in equal } \\
\text { quantity }\end{array}$ & $\begin{array}{l}\text { Apply in the } \\
\text { affected area }\end{array}$ & $\begin{array}{l}\text { Twice a day for } \\
21 \text { days }\end{array}$ \\
\hline & & $\begin{array}{l}\text { Kapur, } \\
\text { Alasi oil }\end{array}$ & $\begin{array}{l}\text { Linum usitatissimum Linn. } \\
\text { (Linaceae) }\end{array}$ & $\begin{array}{l}\text { Grind and gently } \\
\text { boiled with Alasi oil }\end{array}$ & $\begin{array}{l}\text { Lukewarm oil } \\
\text { apply in the } \\
\text { affected area }\end{array}$ & $\begin{array}{l}\text { Twice a day for } \\
4-5 \text { days }\end{array}$ \\
\hline & & $\begin{array}{l}\text { Lahsun (bulb), } \\
\text { Hingu (exude), } \\
\text { Mustard oil }\end{array}$ & $\begin{array}{l}\text { Allium sativum Linn. (Amaryllidaceae); } \\
\text { N.asafetida Falc. Ex Lindl. (Apiaceae); } \\
\text { B.campestris Linn. var. sarson Prain } \\
\text { (Brassicaceae) }\end{array}$ & $\begin{array}{l}\text { Lasun and Hingu } \\
\text { gently boiled in } \\
\text { mustard oil }\end{array}$ & $\begin{array}{l}\text { Lukewarm oil } \\
\text { apply in the } \\
\text { affected area }\end{array}$ & $\begin{array}{l}\text { Twice a day for } \\
4-5 \text { days }\end{array}$ \\
\hline & & $\begin{array}{l}\text { Tambaku (Leaf), } \\
\text { Water }\end{array}$ & $\begin{array}{l}\text { Nicotiana tabacum Linn. } \\
\text { (Solanaceae) }\end{array}$ & $\begin{array}{l}\text { Leaf gently boiled } \\
\text { with water }\end{array}$ & $\begin{array}{l}\text { Lukewarm water } \\
\text { apply in the } \\
\text { affected area }\end{array}$ & $\begin{array}{l}\text { Twice a day for } \\
4-5 \text { days }\end{array}$ \\
\hline & & $\begin{array}{l}\text { Chameli (leaf), } \\
\text { Narial oil }\end{array}$ & $\begin{array}{l}\text { J.grandiflorum Linn. (Oleaceae); } \\
\text { Cocus nucifera L. (Arecaceae) }\end{array}$ & $\begin{array}{l}\text { Leaf gently boiled } \\
\text { with Narial oil }\end{array}$ & $\begin{array}{l}\text { Lukewarm oil } \\
\text { apply in the } \\
\text { affected area }\end{array}$ & $\begin{array}{l}\text { Twice a day for } \\
4-5 \text { days }\end{array}$ \\
\hline & & $\begin{array}{l}\text { Kela (leaf), } \\
\text { Narial (skill), } \\
\text { Kalimirch (seed); } \\
\text { Alasi oil }\end{array}$ & $\begin{array}{l}\text { Musa paradisiaca Linn. (Musaceae); } \\
\text { Cocus nucifera L. (Arecaceae); } \\
\text { Piper nigrum L. (Piperaceae); } \\
\text { Linum usitatissimum Linn. (Linaceae) }\end{array}$ & $\begin{array}{l}\text { Burn the Kela leaf \& } \\
\text { Narial skill to make ash } \\
\text { grind the Kalimirch } \\
\text { to make a powder mix } \\
\text { together \& gently boiled }\end{array}$ & $\begin{array}{l}\text { Lukewarm oil } \\
\text { apply in the } \\
\text { affected area } \\
\text { with Alasi oil }\end{array}$ & $\begin{array}{l}\text { Twice a day for } \\
4-5 \text { days }\end{array}$ \\
\hline & & $\begin{array}{l}\text { Lasun (bulb), } \\
\text { Ajan (seed), } \\
\text { Amarbel (aerial part), } \\
\text { Mustard oil }\end{array}$ & $\begin{array}{l}\text { A.sativum Linn. (Amaryllidaceae); } \\
\text { A.mexicana L. (Papaveraceae); } \\
\text { C.reflexa Roxb. (Convolvulaceae } \\
\text { sensu lato), B.campestris Linn. var. } \\
\text { sarson Prain (Brassicaceae) }\end{array}$ & $\begin{array}{l}\text { Lasun, Ajan and } \\
\text { Amarbel gently boiled } \\
\text { with mustard oil }\end{array}$ & $\begin{array}{l}\text { Lukewarm oil } \\
\text { apply in the } \\
\text { affected area }\end{array}$ & $\begin{array}{l}\text { Twice a day for } \\
4-5 \text { days }\end{array}$ \\
\hline & & $\begin{array}{l}\text { Mahua (leaves), } \\
\text { Hadzod (aerial part) }\end{array}$ & $\begin{array}{l}\text { Madhuca longifolia (Koenig) Macb. } \\
\text { (Sapotaceae); Cissus quandragularis } \\
\text { L. (Vitaceae) }\end{array}$ & $\begin{array}{l}\text { Grind the ingredients } \\
\text { to make the paste }\end{array}$ & $\begin{array}{l}\text { Apply in the } \\
\text { affected area }\end{array}$ & $\begin{array}{l}\text { Twice a day for } \\
5 \text { days }\end{array}$ \\
\hline & & $\begin{array}{l}\text { Ama haldi (rhizome) } \\
\text { Mehandi (root) }\end{array}$ & $\begin{array}{l}\text { Curcuma amada Roxb. (Zingiberaceae); } \\
\text { Lawsonia inermis L. (Lythraceae) }\end{array}$ & $\begin{array}{l}\text { Grind the ingredients } \\
\text { to make the paste }\end{array}$ & $\begin{array}{l}\text { Apply in the } \\
\text { affected area }\end{array}$ & $\begin{array}{l}\text { Twice a day for } \\
5 \text { days }\end{array}$ \\
\hline & & $\begin{array}{l}\text { Satyanashi (leaves); } \\
\text { Kalimirch (fruit) }\end{array}$ & $\begin{array}{l}\text { Argemone mexicana L. (Papaveraceae); } \\
\text { Piper nigrum L. (Piperaceae) }\end{array}$ & $\begin{array}{l}\text { Grind the ingredients } \\
\text { to make the paste }\end{array}$ & $\begin{array}{l}\text { Apply in the } \\
\text { affected area }\end{array}$ & $\begin{array}{l}\text { Twice a day for } \\
5 \text { days }\end{array}$ \\
\hline & & $\begin{array}{l}\text { Sarpunkha (leaves), } \\
\text { Bhatkatia (leaves) }\end{array}$ & $\begin{array}{l}\text { T.purpurea (L.) Pers. (Leguminoseae); } \\
\text { Solanum virginianum L. (Solanaceae) }\end{array}$ & $\begin{array}{l}\text { Grind the ingredients } \\
\text { to make the paste }\end{array}$ & $\begin{array}{l}\text { Apply on the } \\
\text { affected area }\end{array}$ & $\begin{array}{l}\text { Twice a day for } \\
3 \text { days }\end{array}$ \\
\hline
\end{tabular}




\begin{tabular}{|c|c|c|c|c|c|}
\hline & Paras papal (seeds) & Thespesia populnea (Malvaceae) & $\begin{array}{l}\text { Grind the ingredients } \\
\text { to make the paste }\end{array}$ & $\begin{array}{l}\text { Apply on the } \\
\text { affected area }\end{array}$ & $\begin{array}{l}\text { Twice a day for } \\
3 \text { days }\end{array}$ \\
\hline & $\begin{array}{l}\text { Neem (leaf) } \\
\text { Karela (leaf) }\end{array}$ & $\begin{array}{l}\text { Azadirchata indica (Meliaceae), } \\
\text { Momordica charantia (Cucurbitaceae) }\end{array}$ & $\begin{array}{l}\text { Grind the ingredients } \\
\text { to make the paste }\end{array}$ & $\begin{array}{l}\text { Apply on the } \\
\text { affected area }\end{array}$ & $\begin{array}{l}\text { Twice a day for } \\
3 \text { days }\end{array}$ \\
\hline & Bargad (twig) & Ficus benghalensis (Moraceae) & $\begin{array}{l}\text { Grind the ingredients } \\
\text { to make the paste }\end{array}$ & $\begin{array}{l}\text { Apply on the } \\
\text { affected area }\end{array}$ & $\begin{array}{l}\text { Twice a day for } \\
3 \text { days }\end{array}$ \\
\hline 9. Fever & $\begin{array}{l}\text { Nenua (leaves); } \\
\text { Mammari (leaves) }\end{array}$ & $\begin{array}{l}\text { L.cylindrica (L.) Roem. (Cucurbita.); } \\
\text { O.americanum L. (Lamiaceae) }\end{array}$ & $\begin{array}{l}\text { Grind the ingredients } \\
\text { to make the paste }\end{array}$ & $\begin{array}{l}\text { Apply on the } \\
\text { affected area }\end{array}$ & $\begin{array}{l}\text { Twice a day for } \\
3 \text { days }\end{array}$ \\
\hline & $\begin{array}{l}\text { Harsingar (leaf), } \\
\text { Kalesar (leaf), } \\
\text { Kalimirch (fruit) }\end{array}$ & $\begin{array}{l}\text { N.arbortristis L. (Oleaceae); } \\
\text { A.indica Linn. (Aristolochiaceae); } \\
\text { P.nigrum L. (Piperaceae) }\end{array}$ & $\begin{array}{l}\text { Cut into small pieces of } \\
\text { ingredients and boiled } \\
\text { to make a decoction }\end{array}$ & $\begin{array}{l}\text { Decoction is } \\
\text { taken with } \\
\text { water }\end{array}$ & $\begin{array}{l}40-50 \mathrm{ml} \text { twice a } \\
\text { day for 3-5 days }\end{array}$ \\
\hline & $\begin{array}{l}\text { Tulsi (leaf), } \\
\text { Kalimirch (fruit), } \\
\text { Kalesar (leaf) }\end{array}$ & $\begin{array}{l}\text { O.tenuiflorum L. (Lamiaceae); } \\
\text { P.nigrum L. (Piperaceae); } \\
\text { A.indica Linn.(Aristolochiaceae) }\end{array}$ & $\begin{array}{l}\text { Cut into small pieces of } \\
\text { ingredients and boiled } \\
\text { to make a decoction }\end{array}$ & $\begin{array}{l}\text { Decoction is } \\
\text { taken with } \\
\text { water }\end{array}$ & $\begin{array}{l}40-50 \mathrm{ml} \text { twice a } \\
\text { day for 3-5 days }\end{array}$ \\
\hline & $\begin{array}{l}\text { Saherua (leaf), } \\
\text { Kalesar (leaf), } \\
\text { Neem (twig) }\end{array}$ & $\begin{array}{l}\text { N.arbortristis L. (Oleaceae); } \\
\text { A.indica Linn. (Aristolochiaceae); } \\
\text { A.indica A. Juss. (Meliaceae) }\end{array}$ & $\begin{array}{l}\text { Cut into small pieces of } \\
\text { ingredients and boiled } \\
\text { to make a decoction }\end{array}$ & $\begin{array}{l}\text { Decoction is } \\
\text { taken with } \\
\text { water }\end{array}$ & $\begin{array}{l}40-50 \mathrm{ml} \text { twice a } \\
\text { day for 3-5 days }\end{array}$ \\
\hline & $\begin{array}{l}\text { Guduchi (stem), } \\
\text { Kalimirch (seed), } \\
\text { Tulsi (leaves), } \\
\text { Kalmegh (Panchanga) }\end{array}$ & $\begin{array}{l}\text { T.cordifolia (Willd.) Miers ex Hook. } \\
\text { f. \& Thoms, (Menispermaceae); } \\
\text { Piper nigrum L. (Piperaceae); } \\
\text { O.tenuiflorum L. (Lamiaceae); } \\
\text { A.paniculata (Burm.f.) Wall. ex } \\
\text { ex. Nees (Acanthaceae) }\end{array}$ & $\begin{array}{l}\text { Cut into small pieces of } \\
\text { ingredients and boiled } \\
\text { and make the decoction }\end{array}$ & $\begin{array}{l}\text { Decoction is } \\
\text { taken with } \\
\text { water }\end{array}$ & $\begin{array}{l}20-30 \mathrm{ml} \text { twice a } \\
\text { day for } 5 \text { days }\end{array}$ \\
\hline & $\begin{array}{l}\text { Kalmegh (Panchang), } \\
\text { Kareli (seed), } \\
\text { Guduchi (stem) }\end{array}$ & $\begin{array}{l}\text { A.paniculata (Burm.f.) Wall.ex Nees. } \\
\text { (Acanthaceae); M.charantia L. } \\
\text { (Cucurbitaceae); T.cordifolia (Willd.) } \\
\text { Miers ex Hook. F. \& Thoms } \\
\text { (Menispermaceee) }\end{array}$ & $\begin{array}{l}\text { Cut into small pieces of } \\
\text { ingredients and boiled } \\
\text { to make a decoction }\end{array}$ & $\begin{array}{l}\text { Decoction is } \\
\text { taken with } \\
\text { water }\end{array}$ & $\begin{array}{l}40-50 \mathrm{ml} \text { twice a } \\
\text { day for 3-5 days }\end{array}$ \\
\hline & $\begin{array}{l}\text { Kalmegh (Panchanga), } \\
\text { Neem (leaves), } \\
\text { Tulsi (leaves) }\end{array}$ & $\begin{array}{l}\text { A.paniculata (Burm.f.) Wall. ex.Nees. } \\
\text { (Acanthaceae); A.indica A.Juss. } \\
\text { (Meliaceae); O.tenuiflorum L. (Lam.) }\end{array}$ & $\begin{array}{l}\text { Cut into small pieces of } \\
\text { ingredients and boiled } \\
\text { to make the decoction }\end{array}$ & $\begin{array}{l}\text { Take the } \\
\text { decoction }\end{array}$ & $\begin{array}{l}10-20 \mathrm{ml} \text { twice a } \\
\text { day for } 5 \text { days }\end{array}$ \\
\hline & $\begin{array}{l}\text { Kalesar (leaf), } \\
\text { Pippali (fruit), } \\
\text { Kalimirch (fruit), } \\
\text { Karanj (seed), } \\
\text { Guduchi (stem) }\end{array}$ & $\begin{array}{l}\text { A.indica Linn. (Aristolochiaceae); } \\
\text { P.nigrum L. (Piperaceae); P.longum L. } \\
\text { (Piperaceae); M.charantia L. } \\
\text { (Cucurbitaceae); T.cordifolia (Willd.) } \\
\text { Miers ex Hook. F. \& Thoms (Menisperm }\end{array}$ & $\begin{array}{l}\text { Cut into small pieces of } \\
\text { ingredients and boiled } \\
\text { to make a decoction } \\
\text { aceae) }\end{array}$ & $\begin{array}{l}\text { Decoction is } \\
\text { taken with } \\
\text { water }\end{array}$ & $\begin{array}{l}40-50 \mathrm{ml} \text { twice a } \\
\text { day for 3-5 days }\end{array}$ \\
\hline $\begin{array}{l}\text { 10. Malaria } \\
\text { fever }\end{array}$ & $\begin{array}{l}\text { Nari damdari, } \\
\text { Neem ghetua, } \\
\text { Tulsi (leaves), } \\
\text { Kalimirch }\end{array}$ & $\begin{array}{l}\text { E.axillare (Poir. ex Lam.) A. Raynal } \\
\text { (Gentianaceae); A.india, A.Juss. } \\
\text { (Meliaceae); O.tenuiflorum L. } \\
\text { (Lamiaceae) P.nigrum L. (Piperaceae) }\end{array}$ & $\begin{array}{l}\text { Cut into small pieces of } \\
\text { ingredients and boiled } \\
\text { to make the decoction }\end{array}$ & $\begin{array}{l}\text { Decoction is } \\
\text { taken with } \\
\text { water }\end{array}$ & $\begin{array}{l}10-20 \mathrm{ml} \text { twice a } \\
\text { day for } 5 \text { days }\end{array}$ \\
\hline & $\begin{array}{l}\text { Sheesham (leaves), } \\
\text { Kalimirch (fruit), Honey }\end{array}$ & $\begin{array}{l}\text { Dalbergia sissoo D.C. (Leguminosae); } \\
\text { Piper nigrum L. (Piperaceae) }\end{array}$ & $\begin{array}{l}\text { Grind and make the } \\
\text { churna }\end{array}$ & $\begin{array}{l}\text { Churna is taken } \\
\text { with honey }\end{array}$ & $\begin{array}{l}2 \text { gm churna } \\
\text { twice a day for } \\
7 \text { days }\end{array}$ \\
\hline & $\begin{array}{l}\text { Van jeera (seeds), } \\
\text { Tulsi (leaf) }\end{array}$ & $\begin{array}{l}\text { Baccharoides anthelmintica (Aster.), } \\
\text { Ocimum sanctum (Lamiaceae) }\end{array}$ & $\begin{array}{l}\text { Cut into small pieces of } \\
\text { ingredients and boiled } \\
\text { to make the decoction }\end{array}$ & $\begin{array}{l}\text { Decoction is } \\
\text { taken with } \\
\text { water }\end{array}$ & $\begin{array}{l}10-20 \mathrm{ml} \text { twice a } \\
\text { day for } 5 \text { days }\end{array}$ \\
\hline & $\begin{array}{l}\text { Van jeera (seeds); } \\
\text { Neem (leaf) }\end{array}$ & $\begin{array}{l}\text { B.anthelmintica (Asteraceae), } \\
\text { Azadirachta indica (Meliaceae) }\end{array}$ & $\begin{array}{l}\text { Cut into small pieces of } \\
\text { ingredients and boiled } \\
\text { to make the decoction }\end{array}$ & $\begin{array}{l}\text { Decoction is } \\
\text { taken with } \\
\text { water }\end{array}$ & $\begin{array}{l}10-20 \mathrm{ml} \text { twice a } \\
\text { day for } 5 \text { days }\end{array}$ \\
\hline & Kala bach (root) & $\begin{array}{l}\text { Hymenodictyon orixense } \\
\text { (Rubiaceae) }\end{array}$ & $\begin{array}{l}\text { Cut into small pieces of } \\
\text { ingredients and boiled } \\
\text { to make the decoction }\end{array}$ & $\begin{array}{l}\text { Decoction is } \\
\text { taken with } \\
\text { water }\end{array}$ & $\begin{array}{l}10-20 \mathrm{ml} \text { twice a } \\
\text { day for } 5 \text { days }\end{array}$ \\
\hline & $\begin{array}{l}\text { Peacock (feather), } \\
\text { Jivanti Honey }\end{array}$ & $\begin{array}{l}\text { Leptadenia reticulata } \mathrm{W} \& \mathrm{~A} . \\
\text { (Asclepiadaceae) }\end{array}$ & $\begin{array}{l}\text { Burn the feather to } \\
\text { make a ash }\end{array}$ & $\begin{array}{l}\text { Powder is taken } \\
\text { with honey }\end{array}$ & $\begin{array}{l}1-2 \text { gm powder } \\
\text { twice a day for } \\
1-2 \text { days }\end{array}$ \\
\hline 11. Vomiting & $\begin{array}{l}\text { Lavanga (flower bud), } \\
\text { water }\end{array}$ & $\begin{array}{l}\text { Syzygium aromaticum (L.) Merr. } \\
\text { \& L.M. Perry. (Myrtaceae) }\end{array}$ & $\begin{array}{l}\text { Boil the water with } \\
\text { Lavanga flower bud }\end{array}$ & Taken as such & $\begin{array}{l}2-3 \text { liters in } \\
\text { day for } 3-5 \text { days }\end{array}$ \\
\hline & $\begin{array}{l}\text { Onion (bulb), } \\
\text { Lalmirch (seed), Water }\end{array}$ & $\begin{array}{l}\text { Allium cepa Linn. (Amaryllidaceae); } \\
\text { Capsicum annuum L. (Solanaceae) }\end{array}$ & $\begin{array}{l}\text { Grind the bulb \& Mirch } \\
\text { seeds \& make solution }\end{array}$ & Taken as such & $\begin{array}{l}30-40 \mathrm{ml} \text { twice a } \\
\text { day for } 3 \text { days }\end{array}$ \\
\hline & $\begin{array}{l}\text { Lalmirch (seed), } \\
\text { Onion (bulb), } \\
\text { Ajwan (fruit) }\end{array}$ & $\begin{array}{l}\text { C.annuum L. (Solanaceae); A.cepa } \\
\text { Linn. (Amaryllidaceae); T.ammi (L.) } \\
\text { Sprague (Apiaceae); }\end{array}$ & $\begin{array}{l}\text { Grind all ingredients to } \\
\text { make a paste and } \\
\text { dissolve in water } \\
\text { to make a solution }\end{array}$ & Taken as such & $\begin{array}{l}30-40 \mathrm{ml} \text { twice a } \\
\text { day for } 3 \text { days }\end{array}$ \\
\hline & $\begin{array}{l}\text { Lavanga (flower), } \\
\text { Onion (bulb), } \\
\text { Ajwain (fruit), } \\
\text { Honey }\end{array}$ & $\begin{array}{l}\text { S.aromaticum (L.) Merr. \& L.M. } \\
\text { Perry. (Myrtaceae), A.cepa Linn. } \\
\text { (Amaryllidaceae); T.ammi (L.) } \\
\text { Sprague (Apiaceae) }\end{array}$ & $\begin{array}{l}\text { Grind all ingredients } \\
\text { to make a paste }\end{array}$ & $\begin{array}{l}\text { Paste is taken } \\
\text { with honey }\end{array}$ & $\begin{array}{l}1-2 \text { gm twice a } \\
\text { day for } 1-2 \text { days }\end{array}$ \\
\hline & $\begin{array}{l}\text { Sugar, } \\
\text { Namak, Water }\end{array}$ & & Make a solution & Taken as such & $\begin{array}{l}\text { 2-3 liters in } \\
\text { day for 3-5 days }\end{array}$ \\
\hline & $\begin{array}{l}\text { Donda (badi elaichi), } \\
\text { Honey }\end{array}$ & $\begin{array}{l}\text { Amomum subulatum Roxb. } \\
\text { (Zingiberaceae) }\end{array}$ & $\begin{array}{l}\text { Grind the Donda to } \\
\text { make a powder }\end{array}$ & $\begin{array}{l}\text { Powder is taken } \\
\text { with honey }\end{array}$ & $\begin{array}{l}2 \text { gm powder } \\
\text { twice a day for } \\
1-3 \text { days }\end{array}$ \\
\hline
\end{tabular}




\begin{tabular}{|c|c|c|c|c|c|}
\hline & $\begin{array}{l}\text { Bahera (stem bark), } \\
\text { Honey }\end{array}$ & $\begin{array}{l}\text { Terminalia bellirica (Breyn ex Gaertn.) } \\
\text { Roxb. (Combretaceae) }\end{array}$ & $\begin{array}{l}\text { Grind the ingredient } \\
\text { to make a powder }\end{array}$ & $\begin{array}{l}\text { Powder is taken } \\
\text { with honey }\end{array}$ & $\begin{array}{l}1-2 \text { gm powder } \\
\text { twice a day for } \\
1-3 \text { days }\end{array}$ \\
\hline \multirow[t]{15}{*}{$\begin{array}{l}\text { 12. Loose } \\
\text { motion }\end{array}$} & $\begin{array}{l}\text { Mango (ripe fruit), } \\
\text { milk }\end{array}$ & $\begin{array}{l}\text { Mangifera indica Linn. } \\
\text { (Anacardiaceae) }\end{array}$ & $\begin{array}{l}\text { Extract the juice of } \\
\text { ripe fruit mixed with } \\
\text { milk to make a solution }\end{array}$ & Taken as such & $\begin{array}{l}100 \mathrm{ml} \text { twice a } \\
\text { day for } 1-2 \text { days }\end{array}$ \\
\hline & $\begin{array}{l}\text { Ajwain (fruit), } \\
\text { Onion (bulb), } \\
\text { Methi (seed), water }\end{array}$ & $\begin{array}{l}\text { T.ammi (L.) Sprague (Apiaceae); } \\
\text { Allium cepa Linn. (Amaryllidaceae); } \\
\text { T.foenumgraecum Linn. (Fabaceae) }\end{array}$ & $\begin{array}{l}\text { Grind all ingredients } \\
\text { to make a solution }\end{array}$ & Taken as such & $\begin{array}{l}100 \mathrm{ml} \text { thrice a } \\
\text { day for } 1-3 \text { days }\end{array}$ \\
\hline & Duddhiya (leaf) & $\begin{array}{l}\text { Euphorbia neriifolia Linn. } \\
\text { (Euphorbiaceae) }\end{array}$ & Extract the juice & Taken as such & $\begin{array}{l}10-20 \mathrm{ml} \text { twice a } \\
\text { day for one day }\end{array}$ \\
\hline & $\begin{array}{l}\text { Kaitha (leaf), } \\
\text { Curd }\end{array}$ & $\begin{array}{l}\text { Feronia limonia (L.) Swingle } \\
\text { (Rutaceae) }\end{array}$ & $\begin{array}{l}\text { Extract the juice of } \\
\text { leaves \& mix with curd }\end{array}$ & Taken as such & $\begin{array}{l}40-50 \mathrm{ml} \text { twice a } \\
\text { day for } 1-2 \text { days }\end{array}$ \\
\hline & $\begin{array}{l}\text { Patharchata (root), } \\
\text { Milk }\end{array}$ & $\begin{array}{l}\text { Boerhavia diffusa } \mathrm{L} . \\
\text { (Nyctaginaceae) }\end{array}$ & $\begin{array}{l}\text { Extract the juice of } \\
\text { root \& mix with milk }\end{array}$ & Taken as such & $\begin{array}{l}40-50 \mathrm{ml} \text { twice } \mathrm{a} \\
\text { day for } 1-2 \text { days }\end{array}$ \\
\hline & $\begin{array}{l}\text { Bel (fruit pulp), } \\
\text { Kaitha (leaf), Water }\end{array}$ & $\begin{array}{l}\text { A.marmelos Linn. Corr. (Rutaceae), } \\
\text { F.limonia (L.) Swingle (Rutaceae) }\end{array}$ & $\begin{array}{l}\text { Grind the both ingredi- } \\
\text { ents to make a solution }\end{array}$ & Take the solution & $\begin{array}{l}100 \mathrm{ml} \text { thrice a } \\
\text { day for } 1-2 \text { days }\end{array}$ \\
\hline & $\begin{array}{l}\text { Ajwain (fruit), } \\
\text { Lavanga (flower bud), } \\
\text { Kalimirch (seed) }\end{array}$ & $\begin{array}{l}\text { T.ammi (L.) Sprague (Apiaceae); } \\
\text { S.aromaticum (L.) Merr. \& L.M. } \\
\text { Perry. (Myrtaceae); Piper nigrum L. } \\
\text { (Piperaceae) }\end{array}$ & $\begin{array}{l}\text { Grind to make } \\
\text { a powder }\end{array}$ & $\begin{array}{l}\text { Power is } \\
\text { taken with water }\end{array}$ & $\begin{array}{l}1-2 \text { gm powder } \\
\text { twice a day } \\
\text { for } 1-2 \text { days }\end{array}$ \\
\hline & Sheesham (leaves), Curd & $\begin{array}{l}\text { Dalbergia sissoo DC. } \\
\text { (Leguminosae) }\end{array}$ & Extract the juice & Taken as such & $\begin{array}{l}10-20 \mathrm{ml} \text { twice a } \\
\text { day for one day }\end{array}$ \\
\hline & $\begin{array}{l}\text { Methi (Seed, } \\
\text { kachhi \& Pakki), } \\
\text { Ajwain (fruit) }\end{array}$ & $\begin{array}{l}\text { Trigonella foenumgraecum Linn. } \\
\text { (Fabaceae) }\end{array}$ & $\begin{array}{l}\text { Grind to make a } \\
\text { powder }\end{array}$ & $\begin{array}{l}\text { Powder is taken } \\
\text { with water }\end{array}$ & $\begin{array}{l}1-2 \text { gm powder } \\
\text { twice a day for } \\
1-2 \text { days }\end{array}$ \\
\hline & $\begin{array}{l}\text { Babul (leaves), } \\
\text { Sugar, Water }\end{array}$ & $\begin{array}{l}\text { Vachellia nilotica (L.) P.J.H. Hurter } \\
\& \text { Mabb. (Leguminosae) }\end{array}$ & $\begin{array}{l}\text { Grind the both ingredi- } \\
\text { ents to make a solution }\end{array}$ & Take the solution & $\begin{array}{l}100 \mathrm{ml} \text { thrice a } \\
\text { day for } 1-2 \text { days }\end{array}$ \\
\hline & Silahatta (leaves) & $\begin{array}{l}\text { Bergenia ciliata (Haw.) Sternb. } \\
\text { (Saxifragaceae) }\end{array}$ & Extract the juice & Taken as such & $\begin{array}{l}10-20 \mathrm{ml} \text { twice a } \\
\text { day for one day }\end{array}$ \\
\hline & Kudo (leaves) & $\begin{array}{l}\text { Holarrhena antidysenterica } \\
\text { (Apocynacea) }\end{array}$ & Extract the juice & Taken as such & $\begin{array}{l}10-20 \mathrm{ml} \text { twice a } \\
\text { day for one day }\end{array}$ \\
\hline & Maida (stem bark) & $\begin{array}{l}\text { Litsea glutinosa } \\
\text { (Lauraceae) }\end{array}$ & $\begin{array}{l}\text { Grind the ingredient } \\
\text { to make a solution }\end{array}$ & Take the solution & $\begin{array}{l}100 \mathrm{ml} \text { thrice a } \\
\text { day for } 1-2 \text { days }\end{array}$ \\
\hline & Sarai/Saal (Raal) & $\begin{array}{l}\text { Shorea robusta } \\
\text { (Dipterocarpaceae) }\end{array}$ & $\begin{array}{l}\text { Grind and make a } \\
\text { powder }\end{array}$ & Take it & $\begin{array}{l}1-2 \text { gm powder } \\
\text { with water twice } \\
\text { a day for } 3 \text { days }\end{array}$ \\
\hline & $\overline{\text { Padin (root) }}$ & $\begin{array}{l}\text { Cissampelos pareira } \\
\text { (Menispermaceae) }\end{array}$ & $\begin{array}{l}\text { Grind the ingredient } \\
\text { to make a solution }\end{array}$ & Take the solution & $\begin{array}{l}100 \mathrm{ml} \text { thrice a } \\
\text { day for } 1-2 \text { days }\end{array}$ \\
\hline \multirow[t]{5}{*}{$\begin{array}{l}\text { 13. Tooth- } \\
\text { eruption }\end{array}$} & Adusa (bark) & $\begin{array}{l}\text { Justicia adhatoda L. } \\
\text { (Acanthaceae) }\end{array}$ & Grind to make a paste & $\begin{array}{l}\text { Paste is apply in } \\
\text { the affected area }\end{array}$ & $\begin{array}{l}\text { Thrice a day for } \\
1-3 \text { days }\end{array}$ \\
\hline & Sitaphala (leaves) & $\begin{array}{l}\text { Annona squamosa } \mathrm{L} . \\
\text { (Annonaceae) }\end{array}$ & $\begin{array}{l}\text { Grind and make the } \\
\text { decoction and gargle }\end{array}$ & Use as such & $\begin{array}{l}20-30 \mathrm{ml} \text { Twice a } \\
\text { day for } 3 \text { days }\end{array}$ \\
\hline & Lehsun (bulb hair) & $\begin{array}{l}\text { Allium sativum } \\
\text { (Amaryllidaceae) }\end{array}$ & Grind to make a paste & $\begin{array}{l}\text { Paste is apply in } \\
\text { the affected area }\end{array}$ & $\begin{array}{l}\text { Thrice a day for } \\
1-3 \text { days }\end{array}$ \\
\hline & Chandrajot (twig) & $\begin{array}{l}\text { Jatropha curcas } \\
\text { (Euphorbiaceae) }\end{array}$ & Grind to make a paste & $\begin{array}{l}\text { Paste is apply in } \\
\text { the affected area }\end{array}$ & $\begin{array}{l}\text { Thrice a day for } \\
1-3 \text { days }\end{array}$ \\
\hline & Akona (latex) & $\begin{array}{l}\text { Calotropis procera } \\
\text { (Asclepiadaceae) }\end{array}$ & & $\begin{array}{l}\text { Apply the latex in } \\
\text { the affected area }\end{array}$ & $\begin{array}{l}\text { Thrice a day for } \\
1-3 \text { days }\end{array}$ \\
\hline $\begin{array}{l}\text { 14. Dislo- } \\
\text { cation of } \\
\text { shoulder }\end{array}$ & Arand oil & $\begin{array}{l}\text { Ricinus communis Linn. } \\
\text { (Euphorbiaceae) }\end{array}$ & & $\begin{array}{l}\text { Massage the oil } \\
\text { into the shoulder }\end{array}$ & $\begin{array}{l}\text { Twice a day for } \\
1-2 \text { days }\end{array}$ \\
\hline \multirow[t]{7}{*}{$\begin{array}{l}\text { 15. Pneu- } \\
\text { monia }\end{array}$} & Ghunghchi (fruit) & $\begin{array}{l}\text { Abrus precatorius } \mathrm{L} . \\
\text { (Leguminosae) }\end{array}$ & $\begin{array}{l}1 / 2-1 \text { fruit grind to make } \\
\text { a paste \& mix with milk }\end{array}$ & Take the solution & $\begin{array}{l}1-2 \text { spoon twice } \\
\text { a day for } 3 \text { days }\end{array}$ \\
\hline & $\begin{array}{l}\text { Hingu (exude), } \\
\text { Lahsun (bulb), } \\
\text { Mustard oil }\end{array}$ & $\begin{array}{l}\text { N.asafoetida Falc. ex Lin. (Apiaceae); } \\
\text { A.sativum Linn. (Amaryllidaceae); } \\
\text { Brassica campestris Linn. Var. sarson } \\
\text { Prain (Brassicaceae) }\end{array}$ & $\begin{array}{l}\text { Boil the both } \\
\text { ingredients in mustard } \\
\text { oil }\end{array}$ & $\begin{array}{l}\text { Massage with } \\
\text { lukewarm oil }\end{array}$ & $\begin{array}{l}\text { Thrice a day for } \\
3-5 \text { days }\end{array}$ \\
\hline & Kukraundha (leaf) & $\begin{array}{l}\text { Blumea lacera (Burm.f) DC. } \\
\text { (Asteraceae) }\end{array}$ & Extract the juice & Taken as such & $\begin{array}{l}10-20 \mathrm{ml} \text { twice a } \\
\text { day for one day }\end{array}$ \\
\hline & $\begin{array}{l}\text { Kukraundha (leaf), } \\
\text { Hingu (exude), } \\
\text { Sunthi (rhizome) }\end{array}$ & $\begin{array}{l}\text { B.lacera (Burm.f) DC. (Asteraceae); } \\
\text { N.asafoetida Falc. ex Lin.. (Apiaceae); } \\
\text { Z.officinale Rosc. (Zingiberaceae) }\end{array}$ & $\begin{array}{l}\text { Extract the juice \& mix } \\
\text { with Hingu \& Sunthi } \\
\text { and gently warmed }\end{array}$ & Apply the liquid & $\begin{array}{l}1-2 \text { spoon twice a } \\
\text { day for } 3 \text { days \& } \\
\text { massage in body }\end{array}$ \\
\hline & $\begin{array}{l}\text { Sehund (leaves), } \\
\text { Hingu (exude), } \\
\text { Lahsun (bulb) }\end{array}$ & $\begin{array}{l}\text { E.neriifolia Linn. (Euphorbiaceae); } \\
\text { N.asafetida Falc. ex Lindl. (Apiaceae); } \\
\text { Allium sativum Linn. (Amaryllidaceae) }\end{array}$ & $\begin{array}{l}\text { Extract the juice \& mix } \\
\text { with Hingu \& Lasun } \\
\text { and gently warmed }\end{array}$ & Apply the liquid & $\begin{array}{l}1-2 \text { spoon twice a } \\
\text { day for } 3 \text { days } \& \\
\text { massage in body }\end{array}$ \\
\hline & $\begin{array}{l}\text { Dagdaua (seed), } \\
\text { Hingu, Honey }\end{array}$ & $\begin{array}{l}\text { Cassia fistula Linn. (Caesalpiniaceae); } \\
\text { Narthex asafoetida Falc. ex Linn. } \\
\text { (Apiaceae) }\end{array}$ & $\begin{array}{l}\text { Grind to make a } \\
\text { powder }\end{array}$ & $\begin{array}{l}\text { Powder is given } \\
\text { with honey }\end{array}$ & $\begin{array}{l}1 / 2-2 \text { gm powder } \\
\text { twice a day for } \\
3 \text { days }\end{array}$ \\
\hline & $\begin{array}{l}\text { Babai (root), } \\
\text { Dagdaua (seed), Honey }\end{array}$ & $\begin{array}{l}\text { Ocimum tenuiflorum Linn. (Lamiaceae); } \\
\text { Cassia fistula Linn. (Caesalpiniaceae) }\end{array}$ & $\begin{array}{l}\text { Grind to make a } \\
\text { powder }\end{array}$ & $\begin{array}{l}\text { Powder is given } \\
\text { with honey }\end{array}$ & $\begin{array}{l}1 / 2-2 \text { gm powder } \\
\text { twice a day for } \\
3 \text { days }\end{array}$ \\
\hline
\end{tabular}




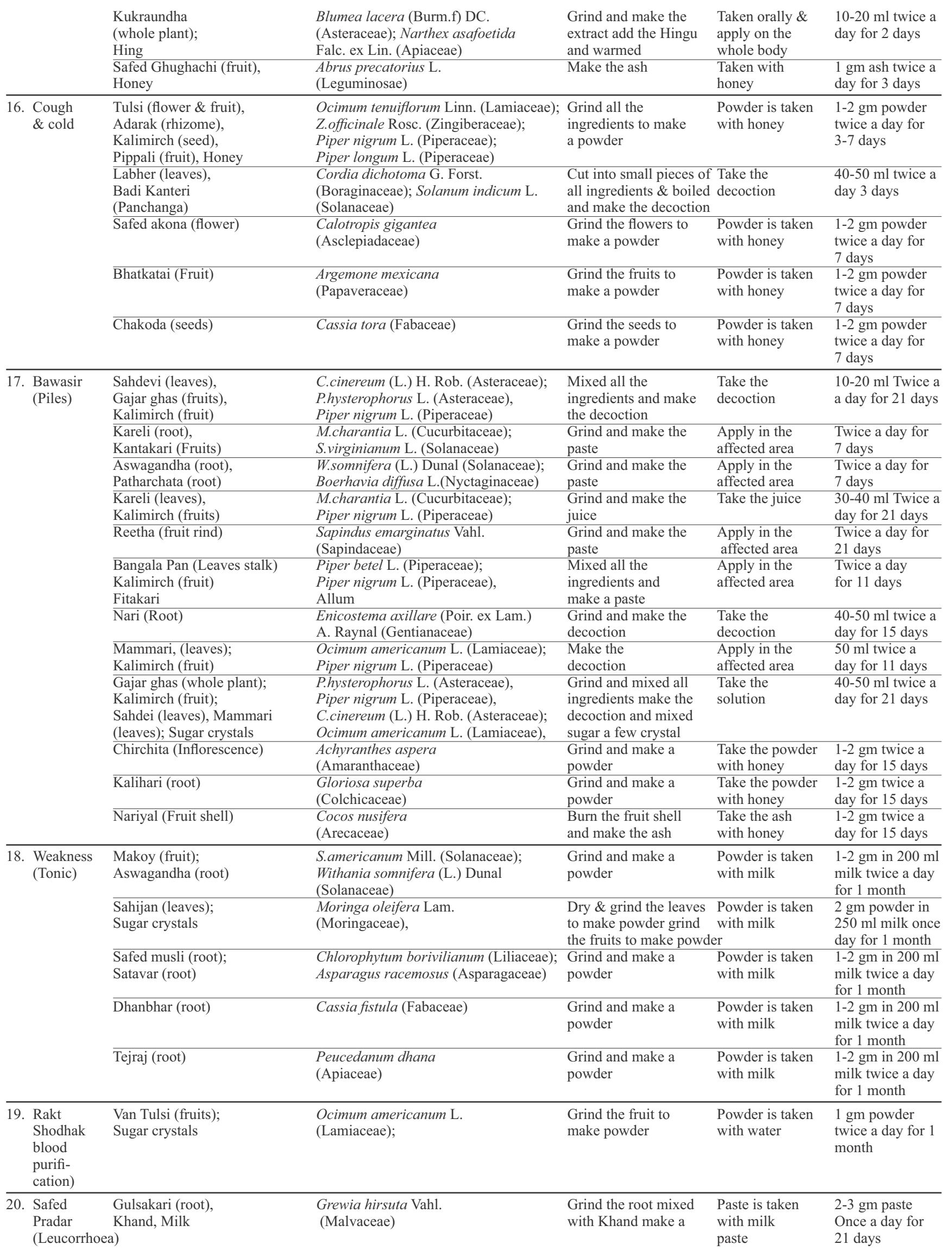




\begin{tabular}{|c|c|c|c|c|c|c|}
\hline 21 & $\begin{array}{l}\text { Rakta- } \\
\text { pradar } \\
\text { (Leucorr- } \\
\text { hoea) }\end{array}$ & $\begin{array}{l}\text { Peepal (Gum), } \\
\text { Elaichi (Fruit), } \\
\text { Khand, Milk } \\
\text { Menda (root bark), } \\
\text { Kamalgatta }\end{array}$ & $\begin{array}{l}\text { Ficus religiosa L. (Moraceae); } \\
\text { Elettaria cardamomum L. Maton } \\
\text { (Zingiberaceae) } \\
\text { Litsea glutinosa (Lour.) Robins. } \\
\text { (Lauraceae); Nelumbo nucifera } \\
\text { Gaertn. (Nelumbonaceae) }\end{array}$ & $\begin{array}{l}\text { Grind three ingredient } \\
\text { and make the powder } \\
\begin{array}{l}\text { Grind the ingredients } \\
\text { to make a powder }\end{array}\end{array}$ & $\begin{array}{l}\text { Powder is taken } \\
\text { with goat milk }\end{array}$ & $\begin{array}{l}2 \text { gm powder } \\
\text { Twice a day for } \\
21 \text { days } \\
2-3 \text { gm powder } \\
\text { with } 200 \mathrm{ml} \text { milk } \\
\text { once a day for } 21 \\
\text { days }\end{array}$ \\
\hline 22. & $\begin{array}{l}\text { Anti- } \\
\text { fertility }\end{array}$ & $\begin{array}{l}\text { Kabila (fruit) } \\
\text { Krishna sariva (root), } \\
\text { milk }\end{array}$ & $\begin{array}{l}\text { M.philippiensis (Lam.) Muell-Arg } \\
\text { (Euphorbiaceae); H.indicus (L.) } \\
\text { R.Br. ex. Schult. (Apocynaceae) }\end{array}$ & $\begin{array}{l}\text { Ground and make } \\
\text { the powder }\end{array}$ & $\begin{array}{l}\text { Powder is taken } \\
\text { with milk }\end{array}$ & $\begin{array}{l}1-2 \text { gm powder } \\
\text { twice a day for } 3 \\
\text { months }\end{array}$ \\
\hline \multirow[t]{2}{*}{23.} & $\begin{array}{l}\text { Napun- } \\
\text { shkata } \\
\text { (Sterility) }\end{array}$ & $\begin{array}{l}\text { Shivlingi (Seed), } \\
\text { Aswagandha (root), } \\
\text { Cow milk }\end{array}$ & $\begin{array}{l}\text { Diplocyclos palmatus (L.) C. Jeffery } \\
\text { (Cucurbitaceae) Withania somnifera } \\
\text { (L.) Dunal (Solanaceae) }\end{array}$ & $\begin{array}{l}\text { Grind the ingredients } \\
\text { to make powder }\end{array}$ & $\begin{array}{l}\text { Powder is taken } \\
\text { with milk }\end{array}$ & $\begin{array}{l}1-2 \text { gm powder is } \\
\text { taken with milk } \\
\text { Twice a day for } \\
3 \text { months }\end{array}$ \\
\hline & & $\begin{array}{l}\text { Peepal (leaves), } \\
\text { Misari (Crystal sugar) }\end{array}$ & $\begin{array}{l}\text { Ficus religiosa } \mathrm{L} \text {. } \\
\text { (Moraceae) }\end{array}$ & $\begin{array}{l}\text { Grind the ingredients } \\
\text { make mixture }\end{array}$ & Take the mixture & $\begin{array}{l}100 \mathrm{ml} \text { twice a } \\
\text { day for } 3 \text { months }\end{array}$ \\
\hline \multirow[t]{6}{*}{24.} & Arthritis & $\begin{array}{l}\text { Sahijan (root bark), } \\
\text { Nirgundi (leaves), }\end{array}$ & $\begin{array}{l}\text { Moringa oleifera Lam. (Moringaceae); } \\
\text { Vitex negundo L. (Lamiaceae); }\end{array}$ & Make the decoction & Use as such & $\begin{array}{l}40-50 \mathrm{ml} \text { Twice a } \\
\text { day for } 11 \text { days }\end{array}$ \\
\hline & & Bariyari (leaves) & $\begin{array}{l}\text { Sida acuta } \text { Burm.f. } \\
\text { (Malvaceae) }\end{array}$ & Make the decoction & Use as such & $\begin{array}{l}40-50 \mathrm{ml} \text { Twice a } \\
\text { day for } 11 \text { days }\end{array}$ \\
\hline & & $\begin{array}{l}\text { Kala dhatura (leaves), } \\
\text { Aak (leaves), } \\
\text { Erand (leaves) }\end{array}$ & $\begin{array}{l}\text { D.metel L. (Solanaceae); C.gigantia } \\
\text { (L.); Dryand. (Asclepiadaceae); } \\
\text { Ricinus communis L. (Euphorbiaceae); }\end{array}$ & $\begin{array}{l}\text { Cut the ingredients into } \\
\text { small pieces \& boiled } \\
\text { with Til Taila make oil }\end{array}$ & $\begin{array}{l}\text { Apply on the } \\
\text { affected area }\end{array}$ & $\begin{array}{l}\text { Twice a day for } \\
\text { for } 21 \text { days }\end{array}$ \\
\hline & & $\begin{array}{l}\text { Sehund (leaves), } \\
\text { Nirgundi (leaves), } \\
\text { Til tail (oil) }\end{array}$ & $\begin{array}{l}\text { E.neriifolia L. (Euphorbiaceae); } \\
\text { Vitex negundo L. (Lamiaceae); } \\
\text { Sesamum indicum L. (Pedaliaceae) }\end{array}$ & $\begin{array}{l}\text { Cut the ingredients into } \\
\text { small pieces \& boiled } \\
\text { with Til Taila make oil }\end{array}$ & $\begin{array}{l}\text { Apply on the } \\
\text { affected area }\end{array}$ & $\begin{array}{l}\text { Twice a day for } \\
3 \text { months }\end{array}$ \\
\hline & & $\begin{array}{l}\text { Midaki (leaves), } \\
\text { Til tail }\end{array}$ & $\begin{array}{l}\text { Vitex negundo L. (Lamiaceae); } \\
\text { Sesamum indicum L. (Pedaliaceae) }\end{array}$ & $\begin{array}{l}\text { Cut the ingredients into } \\
\text { small pieces \& boiled } \\
\text { with Til Taila make oil }\end{array}$ & $\begin{array}{l}\text { Apply on the } \\
\text { affected area }\end{array}$ & $\begin{array}{l}\text { Twice a day for } \\
3 \text { months }\end{array}$ \\
\hline & & Shisham (leaves) & Dalbergia sissoo (Fabaceae) & Extract the juice & Take the juice & $30 \mathrm{ml}$ twice day \\
\hline 25. & $\begin{array}{l}\text { Madhu } \\
\text { makkhi } \\
\text { (Bee-Bite) }\end{array}$ & Kankaua (leaves) & $\begin{array}{l}\text { Commelina benghalensis } \mathrm{L} \text {. } \\
\text { (Commelinaceae) }\end{array}$ & Make the paste & $\begin{array}{l}\text { Apply on the } \\
\text { affected area }\end{array}$ & $\begin{array}{l}\text { Thrice a day for } \\
\text { two days }\end{array}$ \\
\hline \multirow[t]{4}{*}{26.} & $\begin{array}{l}\text { Scorpion } \\
\text { poison }\end{array}$ & $\begin{array}{l}\text { Chakouda (leaves), } \\
\text { Jaggari }\end{array}$ & $\begin{array}{l}\text { Senna tora }(\mathrm{L} .) \text { Roxb. } \\
\text { (Leguminosae) }\end{array}$ & $\begin{array}{l}\text { Ground and make the } \\
\text { paste }\end{array}$ & $\begin{array}{l}\text { Apply in affected } \\
\text { area }\end{array}$ & $\begin{array}{l}\text { Twice a day for } \\
3 \text { days }\end{array}$ \\
\hline & sting & $\begin{array}{l}\text { Lalmirch (Panchanga), } \\
\text { Sahad (Honey) }\end{array}$ & $\begin{array}{l}\text { Capsicum annuит L. } \\
\text { (Solanaceae) }\end{array}$ & Make the paste & $\begin{array}{l}\text { Apply in the } \\
\text { affected area }\end{array}$ & $\begin{array}{l}\text { Twice a day for } \\
\text { one day }\end{array}$ \\
\hline & & Madar (root) & $\begin{array}{l}\text { Calotropis procera (Aiton Dryand.) } \\
\text { (Asclepiadaceae) }\end{array}$ & Make the paste & $\begin{array}{l}\text { Direct apply in } \\
\text { the affected area }\end{array}$ & $\begin{array}{l}\text { Twice a day for } \\
2 \text { days }\end{array}$ \\
\hline & & Tinpatiya (root) & $\begin{array}{l}\text { Oxalis corniculata } \mathrm{L} . \\
\text { (Oxiladaceae) }\end{array}$ & $\begin{array}{l}\text { Grind and make } \\
\text { a paste }\end{array}$ & $\begin{array}{l}\text { Apply on the } \\
\text { affected area }\end{array}$ & $\begin{array}{l}\text { Twice a day for } \\
2 \text { days }\end{array}$ \\
\hline \multirow[t]{6}{*}{27.} & Asthma & $\begin{array}{l}\text { Desi madar (Root \&) } \\
\text { leaves), Afeem, } \\
\text { Nariyal (Pericarp) }\end{array}$ & $\begin{array}{l}\text { Calotropis procera (Aiton.) Dryand. } \\
\text { (Asclepiadaceae); P.somniferum L. } \\
\text { (Papaveraceae); Cocus nucifera L. } \\
\text { (Arecaceae) }\end{array}$ & $\begin{array}{l}\text { Dry \& grind two ingre- } \\
\text { dients make a powder } \\
\text { and Nariayal pericorp } \\
\text { burnt make ash mixed }\end{array}$ & $\begin{array}{l}\text { Powder is taken } \\
\text { with honey }\end{array}$ & $\begin{array}{l}1-2 \text { gm powder } \\
\text { twice a day for } \\
7 \text { days }\end{array}$ \\
\hline & & $\begin{array}{l}\text { Labher (fruit); } \\
\text { Kalimirch }\end{array}$ & $\begin{array}{l}\text { C.dichotoma G. Forst. (Boraginaceae); } \\
\text { Piper nigrum L. (Piperaceae) }\end{array}$ & Make the decoction & $\begin{array}{l}\text { Take the } \\
\text { decoction }\end{array}$ & $\begin{array}{l}40-50 \mathrm{ml} \text { twice a } \\
\text { day for } 21 \text { days }\end{array}$ \\
\hline & & Katkatauni (fruit) & $\begin{array}{l}\text { Solanum virginianum } \mathrm{L} . \\
\text { (Solanaceae) }\end{array}$ & $\begin{array}{l}\text { Burnt the fruits to } \\
\text { make ash }\end{array}$ & $\begin{array}{l}\text { Ash is taken } \\
\text { with honey }\end{array}$ & $\begin{array}{l}1-2 \mathrm{gm} \text { ash twice } \\
\text { a day for } 7 \text { days }\end{array}$ \\
\hline & & Bhelwa (fruit) & $\begin{array}{l}\text { Semecarpus anacardium } \\
\text { (Anacardiaceae) }\end{array}$ & Fry the fruit & $\begin{array}{l}\text { Eat the fruit } \\
\text { with milk }\end{array}$ & $\begin{array}{l}4-5 \text { fruits twice a } \\
\text { day for } 30 \text { days }\end{array}$ \\
\hline & & $\begin{array}{l}\text { Anar (fruit), } \\
\text { Laung (flower), } \\
\text { Neem (leaves) }\end{array}$ & $\begin{array}{l}\text { Punica granatum L. (Punicaceae); } \\
\text { Syzygium aromaticum (L.) Merr. } \\
\text { \& L.M. Perry. (Myrtaceae); } \\
\text { Azadirachata indica, A.Juss. } \\
\text { (Meliaceae) }\end{array}$ & $\begin{array}{l}\text { Lavanga flowers keep } \\
\text { in Anar fruit \& put in } \\
\text { the clay pot under the } \\
\text { Nimba tree; when to } \\
\text { make the ash }\end{array}$ & $\begin{array}{l}\text { Ash is taken } \\
\text { with honey }\end{array}$ & $\begin{array}{l}1-2 \text { gm ash } \\
\text { twice a day for } \\
21 \text { days }\end{array}$ \\
\hline & & Arhar (leaves) & Cajanus cajan (L.) Millsp. & Direct eat the leaves & Use as such & $5 \mathrm{~kg}$ once a days \\
\hline \multirow[t]{3}{*}{28.} & $\begin{array}{l}\text { Retension } \\
\text { of }\end{array}$ & $\begin{array}{l}\text { Lajwanti panchanga } \\
\text { (whole plant) }\end{array}$ & $\begin{array}{l}\text { Mimosa pudica L. } \\
\text { (Leguminosae) }\end{array}$ & $\begin{array}{l}\text { Grind and make the } \\
\text { juice }\end{array}$ & Use as such & $\begin{array}{l}200-250 \mathrm{ml} \text { once } \\
\text { a day for one day }\end{array}$ \\
\hline & $\begin{array}{l}\text { placenta } \\
\text { of animal }\end{array}$ & Bans (Bamboo) ((leaves) & $\begin{array}{l}\text { Bambusa bambos (1.) Voss } \\
\text { (Poaceae) }\end{array}$ & Direct eat the leaves & Use as such & $\begin{array}{l}4-5 \mathrm{~kg} \text { once a } \\
\text { day for one day }\end{array}$ \\
\hline & & Dhan (Paddy) (fruit) & Oryza sativa L. (Poaceae) & Direct eat the Dhan & Use as such & $\begin{array}{l}2-3 \mathrm{~kg} \text { once a } \\
\text { day for one day }\end{array}$ \\
\hline \multirow[t]{3}{*}{29.} & $\begin{array}{l}\text { Cut and } \\
\text { wounds }\end{array}$ & $\begin{array}{l}\text { Ghamira (leaves); } \\
\text { Vidhara (leaves) } \\
\text { Elephant creeper }\end{array}$ & $\begin{array}{l}\text { Eclipta prostrata (L.) (Asteraceae); } \\
\text { Argyreia nervosa (Burm.f.) Bojer } \\
\text { (Convolvulaceae) }\end{array}$ & $\begin{array}{l}\text { Mix and grind the } \\
\text { both to make a paste }\end{array}$ & $\begin{array}{l}\text { Apply in the } \\
\text { affected area }\end{array}$ & $\begin{array}{l}\text { Twice a day for } \\
3 \text { days }\end{array}$ \\
\hline & & Katkatuni (fruits) & $\begin{array}{l}\text { Solanum virginianum } \mathrm{L} . \\
\text { (Solanaceae) }\end{array}$ & $\begin{array}{l}\text { Grind and make the } \\
\text { paste }\end{array}$ & Apply the paste & $\begin{array}{l}\text { Twice a day for } \\
5 \text { days }\end{array}$ \\
\hline & & Rohani (stem bark) & $\begin{array}{l}\text { Mallotus phillipensi } \\
\text { (Euphorbiaceae) }\end{array}$ & $\begin{array}{l}\text { Grind and make the } \\
\text { paste }\end{array}$ & Apply the paste & $\begin{array}{l}\text { Twice a day for } \\
5 \text { days }\end{array}$ \\
\hline
\end{tabular}




\begin{tabular}{|c|c|c|c|c|c|}
\hline & Basondara (root) & $\begin{array}{l}\text { Ageratum conyzoides } \\
\text { (Asteraceae) }\end{array}$ & $\begin{array}{l}\text { Grind and make the } \\
\text { the paste }\end{array}$ & Apply the paste & $\begin{array}{l}\text { Twice a day for } \\
5 \text { days }\end{array}$ \\
\hline \multirow[t]{6}{*}{$\begin{array}{l}\text { 30. Pathari } \\
\text { (Stone) }\end{array}$} & Van bhindi (root) & $\begin{array}{l}\text { Abelmoschus moschatus Medik. } \\
\text { (Malvaceae) }\end{array}$ & Make the powder & $\begin{array}{l}\text { Powder is taken } \\
\text { with water }\end{array}$ & $\begin{array}{l}1-2 \text { gm powder } \\
\text { twice a day for } \\
7 \text { days }\end{array}$ \\
\hline & Chakouda (seeds), Honey & $\begin{array}{l}\text { Senna tora }(\mathrm{L} .) \text { Roxb. } \\
\text { (Leguminosae) }\end{array}$ & $\begin{array}{l}\text { Grind and make the } \\
\text { powder }\end{array}$ & $\begin{array}{l}\text { Powder is taken } \\
\text { with honey }\end{array}$ & $\begin{array}{l}1-2 \text { gm powder } \\
\text { twice a day for } \\
7 \text { days }\end{array}$ \\
\hline & $\begin{array}{l}\text { Safed punarnawa (root), } \\
\text { Misari (Crystal sugar), } \\
\text { Milk, }\end{array}$ & $\begin{array}{l}\text { Boerhavia diffusa } \mathrm{L} . \\
\text { (Nyctanginaceae) }\end{array}$ & $\begin{array}{l}\text { Cut the small pieces of } \\
\text { Punarnawa root to } \\
\text { make paste mixed with } \\
\text { misari and milk to } \\
\text { make mixture }\end{array}$ & Take the mixture & $\begin{array}{l}100-200 \mathrm{ml} \text { twice } \\
\text { a day for } 21 \text { days }\end{array}$ \\
\hline & $\begin{array}{l}\text { Punarnawa (Panchanga), } \\
\text { Gokharu (Panchanga) }\end{array}$ & $\begin{array}{l}\text { Boerhavia diffusa L. (Nyctaginaceae); } \\
\text { Tribulus terrestris L. } \\
\text { (Zygophyllaceae) }\end{array}$ & $\begin{array}{l}\text { Cut into small pieces of } \\
\text { all ingredients } \& \text { boiled } \\
\text { to make decoction }\end{array}$ & $\begin{array}{l}\text { Take the } \\
\text { decoction }\end{array}$ & $\begin{array}{l}40-50 \mathrm{ml} \text { twice } \mathrm{a} \\
\text { day for } 21 \text { days }\end{array}$ \\
\hline & $\begin{array}{l}\text { Kala Dana (seeds), } \\
\text { Kamraj (Panchaga), } \\
\text { Khand }\end{array}$ & $\begin{array}{l}\text { I.nil (L.) Roth. (Convolvulaceae); } \\
\text { Selaginella bryopteris Baker } \\
\text { (Selaginellaceae); Sugar }\end{array}$ & Make the decoction & $\begin{array}{l}\text { Take the } \\
\text { decoction }\end{array}$ & $\begin{array}{l}40-50 \mathrm{ml} \text { twice a } \\
\text { day for } 15 \text { days }\end{array}$ \\
\hline & $\begin{array}{l}\text { Indrayan (root), } \\
\text { Kali musali (leaves) }\end{array}$ & $\begin{array}{l}\text { Cirullus colocynthis (L.) Schrad. } \\
\text { (Cucurbitaceae); Curculigo orchioides } \\
\text { Gaertn. (Hypoxidaceae) }\end{array}$ & To make the decoction & $\begin{array}{l}\text { Take the } \\
\text { decoction }\end{array}$ & $\begin{array}{l}\text { 30-40 } \mathrm{ml} \text { twice a } \\
\text { day for } 7 \text { days }\end{array}$ \\
\hline $\begin{array}{l}\text { 31. Kidney } \\
\text { failure }\end{array}$ & $\begin{array}{l}\text { Ashwagandha (leaves), } \\
\text { Milk }\end{array}$ & $\begin{array}{l}\text { Withania somnifera (L.) Dunal } \\
\text { (Solanaceae); }\end{array}$ & $\begin{array}{l}\text { Grind and make the } \\
\text { powder }\end{array}$ & $\begin{array}{l}\text { Powder is taken } \\
\text { with milk }\end{array}$ & $\begin{array}{l}1-2 \text { gm powder } \\
\text { twice a day for } \\
15 \text { days }\end{array}$ \\
\hline \multirow[t]{2}{*}{$\begin{array}{l}\text { 32. Dhat } \\
\text { (Sperma- } \\
\text { torrhoea) }\end{array}$} & $\begin{array}{l}\text { Satauna (root), } \\
\text { Katkatauni (root) }\end{array}$ & $\begin{array}{l}\text { Alotonia scholaris (L.) R. Br. } \\
\text { (Apocynaceae); Solanum virginianum L. } \\
\text { (Solanaceae) }\end{array}$ & $\begin{array}{l}\text { Grind the ingredients } \\
\& \text { mixed with Mishri } \\
\text { to make a paste }\end{array}$ & Taken with milk & $\begin{array}{l}3 \text { gm paste taken } \\
\text { with milk once a } \\
\text { day for } 21 \text { days }\end{array}$ \\
\hline & $\begin{array}{l}\text { Durva (whole plant) } \\
\text { Mishri (Crystal sugar), } \\
\text { Milk }\end{array}$ & $\begin{array}{l}\text { Cynodon dactylon (L.) Pers. } \\
\text { (Poaceae) }\end{array}$ & $\begin{array}{l}\text { Grind the ingredients } \\
\& \text { mixed with Mishri } \\
\text { to make a paste }\end{array}$ & Taken with milk & $\begin{array}{l}2-3 \text { gm paste in } \\
200 \mathrm{ml} \text { once a } \\
\text { day for } 21 \text { days } \\
\end{array}$ \\
\hline $\begin{array}{l}\text { 33. Lakwa } \\
\text { (Paralysis) }\end{array}$ & Harsingar (Leaves) & $\begin{array}{l}\text { Nyctanthes arbortristis L. } \\
\text { (Oleaceae) }\end{array}$ & Make the decoction & $\begin{array}{l}\text { Take the } \\
\text { decoction }\end{array}$ & $\begin{array}{l}40-50 \mathrm{ml} \text { twice a } \\
\text { day for } 21 \text { days }\end{array}$ \\
\hline \multirow[t]{3}{*}{$\begin{array}{l}\text { 34. Safed } \\
\text { dag } \\
\text { (Leprosy) }\end{array}$} & $\begin{array}{l}\text { Kabila (fruit), } \\
\text { Meida (stem bark), } \\
\text { Gorakhmundi (panchanga), } \\
\text { Kutaj (stem bark) }\end{array}$ & $\begin{array}{l}\text { M.philippensis (Lam.) Muell. Arg. } \\
\text { (Euphorbiaceae); L.glutinosa (Lour.) } \\
\text { C. B. Robins (Lauraceae); S.indicus L. } \\
\text { (Asteraceae); Holarrhena pubescens } \\
\text { Wall.ex G. Don (Apocynaceae) }\end{array}$ & $\begin{array}{l}\text { Grind all the } \\
\text { ingredients to make } \\
\text { a paste }\end{array}$ & $\begin{array}{l}\text { Apply in the } \\
\text { affected area }\end{array}$ & $\begin{array}{l}\text { Twice a day for } \\
3 \text { months }\end{array}$ \\
\hline & Kasaundi (Panchanga) & $\begin{array}{l}\text { Senna occidentalis (L.) Link } \\
\text { (Leguminosae) }\end{array}$ & Make the paste & $\begin{array}{l}\text { Apply in affected } \\
\text { affected area }\end{array}$ & $\begin{array}{l}\text { Twice a day for } \\
3 \text { months }\end{array}$ \\
\hline & Saptaparni (Root bark) & $\begin{array}{l}\text { Alstonia scholaris (Roth.) R. Br. } \\
\text { (Apocynaceae) }\end{array}$ & Make the paste & $\begin{array}{l}\text { Apply on the } \\
\text { affected area }\end{array}$ & $\begin{array}{l}\text { Twice a day for } \\
21 \text { days }\end{array}$ \\
\hline $\begin{array}{l}\text { 35. Sno- } \\
\text { phaelia }\end{array}$ & $\begin{array}{l}\text { Nimbu (fruit), } \\
\text { Madar (Dondi), } \\
\text { Meetha soda }\end{array}$ & $\begin{array}{l}\text { Citrus aurantiifolia (Christm.) Swingle } \\
\text { (Rutaceae), Calotropis procera } \\
\text { (Aiton.) Dryand. } \\
\text { (Apocynaceae) }\end{array}$ & $\begin{array}{l}\text { Madar dondi make the } \\
\text { decoction and mixed } \\
\text { Meetha Soda and } \\
\text { ml lemon juice }\end{array}$ & $\begin{array}{l}\text { Take a } \\
\text { decoction }\end{array}$ & $\begin{array}{l}40-50 \mathrm{ml} \text { twice a } \\
\text { day for } 7 \text { days }\end{array}$ \\
\hline $\begin{array}{l}\text { 36. Foda } \\
\text { funsi } \\
\text { (Pimples) }\end{array}$ & Erandi (leaves) & $\begin{array}{l}\text { Ricinus communis L. } \\
\text { (Euphorbiaceae) }\end{array}$ & $\begin{array}{l}\text { Grind and make a } \\
\text { paste }\end{array}$ & $\begin{array}{l}\text { Apply in the } \\
\text { affected area }\end{array}$ & $\begin{array}{l}\text { Twice a day for } \\
3 \text { months }\end{array}$ \\
\hline $\begin{array}{l}\text { 37. Hydro- } \\
\text { cele }\end{array}$ & $\begin{array}{l}\text { Kantakari (root), } \\
\text { Kalimirch (seed) }\end{array}$ & $\begin{array}{l}\text { Solanum virginianum L. (Solanaceae); } \\
\text { Piper nigrum L. (Piperaceae) }\end{array}$ & $\begin{array}{l}\text { Grind and make the } \\
\text { mixture }\end{array}$ & $\begin{array}{l}\text { Take the } \\
\text { mixture }\end{array}$ & $\begin{array}{l}50-60 \mathrm{ml} \text { twice a } \\
\text { day for } 7 \text { days }\end{array}$ \\
\hline $\begin{array}{l}\text { 38. Blind- } \\
\text { ness of } \\
\text { one eye }\end{array}$ & Gudhal ((leaves) & $\begin{array}{l}\text { Hibiscus rosa-sinensis L. } \\
\text { (Malvaceae) }\end{array}$ & $\begin{array}{l}\text { Grind and make a } \\
\text { juice }\end{array}$ & Use as such & $\begin{array}{l}4-5 \text { drops, apply } \\
\text { in to eyes twice } \\
\text { a day for } 7 \text { days }\end{array}$ \\
\hline \multirow[t]{2}{*}{$\begin{array}{l}\text { 39. } \text { Mirgi } \\
\text { (Epilepsy) }\end{array}$} & Kasaundi (leaves) & $\begin{array}{l}\text { Senna occidentalis (L.) Link } \\
\text { (Leguminosae) }\end{array}$ & $\begin{array}{l}\text { Grind and make a } \\
\text { juice }\end{array}$ & Use as such & $\begin{array}{l}40-50 \mathrm{ml} \text { twice a } \\
\text { day for } 3 \text { months }\end{array}$ \\
\hline & Gummi (Panchanga) & $\begin{array}{l}\text { Leucas cephalotes (Roth.) Spreng. } \\
\text { (Lamiaceae) }\end{array}$ & Make the decoction & Use as such & $\begin{array}{l}40-50 \mathrm{ml} \text { twice a } \\
\text { day for } 3 \text { months }\end{array}$ \\
\hline $\begin{array}{l}\text { 40. Retention } \\
\text { of } \\
\text { Anus, } \\
\text { Guda } \\
\text { nikalna }\end{array}$ & Lajwanti (leaves) & $\begin{array}{l}\text { Mimosa pudica } \mathrm{L} . \\
\text { (Leguminosae) }\end{array}$ & $\begin{array}{l}\text { Grind and make } \\
\text { the paste }\end{array}$ & $\begin{array}{l}\text { Apply in the } \\
\text { affected area }\end{array}$ & $\begin{array}{l}\text { Twice a day for } \\
5 \text { days }\end{array}$ \\
\hline $\begin{array}{l}\text { 41. Pechis } \\
\text { (Dysent- } \\
\text { ery) }\end{array}$ & $\begin{array}{l}\text { Babool (leaves), } \\
\text { Jamun (leaves), } \\
\text { Dhataki (flower), } \\
\text { Kaitha (leaves) }\end{array}$ & $\begin{array}{l}\text { V.nilotica (L.) P.J.H. Hurter \& Mabb. } \\
\text { (Leguminosae); S.cumini (L.) Skeels, } \\
\text { (Myrtaceae); Woodfordia fruticosa (L.) } \\
\text { Kurz. (Lythraceae); Feronia limonia } \\
\text { (L.) Swingle (Rutaceae) }\end{array}$ & $\begin{array}{l}\text { Grind all the } \\
\text { ingredients to make } \\
\text { a mixture }\end{array}$ & $\begin{array}{l}\text { Take a } \\
\text { mixture }\end{array}$ & $\begin{array}{l}\text { 50-60 } \mathrm{ml} \text { twice a } \\
\text { day for } 3 \text { days }\end{array}$ \\
\hline
\end{tabular}




\begin{tabular}{|c|c|c|c|c|c|c|}
\hline 42 & $\begin{array}{l}\text { Gas } \\
\text { (Acidity) }\end{array}$ & $\begin{array}{l}\text { Kaccha Suhaga, Sunthi } \\
\text { (Rizome), Hingu (Excude), } \\
\text { Sahijan (stem bark), } \\
\text { Kala namak }\end{array}$ & $\begin{array}{l}\text { Z.officinale Rosc. (Zingiberaceae); } \\
\text { N.asafoetida Falc. ex Lin. (Apiaceae); } \\
\text { Moringa oleifera Lam. } \\
\text { (Moringaceae) }\end{array}$ & $\begin{array}{l}\text { Grind all the } \\
\text { ingredients to make } \\
\text { a churna }\end{array}$ & $\begin{array}{l}\text { Powder is } \\
\text { taken with } \\
\text { water }\end{array}$ & $\begin{array}{l}1-2 \text { gm twice a } \\
\text { day-15 for days }\end{array}$ \\
\hline & & $\begin{array}{l}\text { Kala Dana (seeds), } \\
\text { Ajowan (fruit) } T\end{array}$ & $\begin{array}{l}\text { I.nil (L.) Roth. (Convolvulaceae); } \\
\text { T.ammi (L.) Sprague (Apiaceae) }\end{array}$ & $\begin{array}{l}\text { Grind and make } \\
\text { the churna }\end{array}$ & $\begin{array}{l}\text { Powder is taken } \\
\text { with warm water }\end{array}$ & $\begin{array}{l}1-2 \text { gm twice a } \\
\text { day for } 21 \text { days }\end{array}$ \\
\hline$\overline{43}$ & Abortion & Bans (root) & $\begin{array}{l}\text { Bambusa bambos (L.) Voss } \\
\text { (Poaceae) }\end{array}$ & $\begin{array}{l}\text { Grind the root to } \\
\text { make a juice }\end{array}$ & Take a juice & $\begin{array}{l}40-50 \mathrm{ml} \text { once a } \\
\text { day for } 3 \text { days }\end{array}$ \\
\hline$\overline{44 .}$ & $\begin{array}{l}\text { Sookha } \\
\text { rog } \\
\text { (Rickets) }\end{array}$ & $\begin{array}{l}\text { Reetha (root), Rakta } \\
\text { punarawa (Panchanga), } \\
\text { Ashwagandha (root), } \\
\text { Adusa (root) }\end{array}$ & $\begin{array}{l}\text { S.emarginatus Vahl. (Sapindaceae); } \\
\text { Boerhavia diffusa L. (Nyctaginaceae); } \\
\text { W.somnifera L. Dunal (Solanaceae); } \\
\text { Justicia adhatoda L. (Acanthaceae) }\end{array}$ & $\begin{array}{l}\text { Cut into small pieces } \\
\text { of all ingredients and } \\
\text { boiled to make the } \\
\text { decoction }\end{array}$ & $\begin{array}{l}\text { Take the } \\
\text { decoction }\end{array}$ & $\begin{array}{l}40-50 \mathrm{ml} \text { twice a } \\
\text { day for } 21 \text { days }\end{array}$ \\
\hline 45. & $\begin{array}{l}\text { Kuposhan } \\
\text { (Malnu- } \\
\text { trition) }\end{array}$ & $\begin{array}{l}\text { Gokchur (Panchang), } \\
\text { Munga (leaves) }\end{array}$ & $\begin{array}{l}\text { Tribulus terrestris L. (Zygophyllaceae); } \\
\text { Moringa oleifera Lam. } \\
\text { (Moringaceae) }\end{array}$ & $\begin{array}{l}\text { Cut and make the } \\
\text { decoction }\end{array}$ & $\begin{array}{l}\text { Take the } \\
\text { decoction }\end{array}$ & $\begin{array}{l}30-40 \mathrm{ml} \text { twice a } \\
\text { day for } 21 \text { days }\end{array}$ \\
\hline 46. & $\begin{array}{l}\text { Head } \\
\text { ache }\end{array}$ & Kala Dana (seeds) & $\begin{array}{l}\text { Ipomoea nil (L.) Roth. } \\
\text { (Convolvulaceae) }\end{array}$ & Make a paste & $\begin{array}{l}\text { Apply in the } \\
\text { affected area }\end{array}$ & 5 days \\
\hline 47. & $\begin{array}{l}\text { Putra } \\
\text { prapti } \\
\text { (Born baby) }\end{array}$ & $\begin{array}{l}\text { Til (seeds), } \\
\text { Kala Dana (root), } \\
\text { Dahi (Curd) }\end{array}$ & $\begin{array}{l}\text { Sesamum indicum L. (Pedaliaceae); } \\
\text { Ipomoea nil (L.) Roth. } \\
\text { (Convolvulaceae) }\end{array}$ & $\begin{array}{l}\text { Grind the ingredients to } \\
\text { make powder \& mixed } \\
\text { with curd and Mishri }\end{array}$ & Use as such & $\begin{array}{l}100 \text { gm orally } \\
\text { taken twice a } \\
\text { day for } 3 \text { months }\end{array}$ \\
\hline$\overline{48 .}$ & Bleeding & $\begin{array}{l}\text { Adusa (Panchanga), } \\
\text { Tinpatiya (root), } \\
\text { Misri (Crystal sugar) }\end{array}$ & $\begin{array}{l}\text { Justicia adhatoda L. (Acanthaceae); } \\
\text { Oxalis corniculata L. } \\
\text { (Oxiladaceae), }\end{array}$ & $\begin{array}{l}\text { Grind the ingredients } \\
\text { to make decoction }\end{array}$ & $\begin{array}{l}\text { Take a } \\
\text { decoction }\end{array}$ & $\begin{array}{l}40-50 \mathrm{ml} \text { one a } \\
\text { day for } 7 \text { days }\end{array}$ \\
\hline \multirow[t]{2}{*}{49.} & \multirow{2}{*}{$\begin{array}{l}\text { Kukur } \\
\text { khansi } \\
\text { (Whoop- } \\
\text { ing Cough) }\end{array}$} & $\begin{array}{l}\text { Makka ke bal (Fruit hair), } \\
\text { Hone }\end{array}$ & Zea mays L. (Poaceae) & $\begin{array}{l}\text { Make the ash of } \\
\text { Makka hairs }\end{array}$ & $\begin{array}{l}\text { Ash is taken } \\
\text { with honey }\end{array}$ & $\begin{array}{l}1-2 \text { gm ash is } \\
\text { taken with honey } \\
\text { twice a day for }\end{array}$ \\
\hline & & $\begin{array}{l}\text { San (leaves), } \\
\text { Saptaparni (leaves), Honey }\end{array}$ & $\begin{array}{l}\text { Crotalaria juncea L. (Leguminosae); } \\
\text { A.scholaris (L.) R.Br. (Apocynaceae) }\end{array}$ & $\begin{array}{l}\text { Grind and make the } \\
\text { ash }\end{array}$ & $\begin{array}{l}\text { Ash is taken } \\
\text { with honey }\end{array}$ & $\begin{array}{l}5 \text { days } \\
2 \text { gm ash is twice } \\
\text { a day for } 3 \text { days }\end{array}$ \\
\hline 50. & $\begin{array}{l}\text { Snake } \\
\text { bite }\end{array}$ & $\begin{array}{l}\text { Padhi (Panchanga), } \\
\text { Gumi (Panchana, } \\
\text { Kali musali (leaves) }\end{array}$ & $\begin{array}{l}\text { C.pareira L. (Menispermaceae); } \\
\text { Leucas cephalotes (Roth) Spreng } \\
\text { (Lamiaceae), Curculigo orchioides } \\
\text { Gaertn. (Hypoxidaceae) }\end{array}$ & $\begin{array}{l}\text { Mix all \& make } \\
\text { decoction }\end{array}$ & $\begin{array}{l}\text { Decoction is } \\
\text { taken orally }\end{array}$ & $\begin{array}{l}40-50 \mathrm{ml} \text { trice a } \\
\text { day for } 2 \text { days }\end{array}$ \\
\hline \multirow[t]{2}{*}{51.} & \multirow[t]{2}{*}{$\begin{array}{l}\text { Adha } \\
\text { shishi } \\
\text { (Migraine) }\end{array}$} & $\begin{array}{l}\text { Tinpatiya (whole plant), } \\
\text { Eranda (leaves), } \\
\text { Baheda ((leaves), } \\
\text { Sheesham (leaves), } \\
\text { Eucalyptus (leaves) }\end{array}$ & $\begin{array}{l}\text { Oxalis corniculata L. (Oxiladaceae); } \\
\text { Ricinus communis L. (Euphorbiaceae); } \\
\text { Terminallia bellirica (Gaertn.) Roxb. } \\
\text { (Combretaceae), Dalbergia sissoo DC. } \\
\text { (Leguminosae); Eucalyptus globulus } \\
\text { Labill. (Myrtaceae) }\end{array}$ & $\begin{array}{l}\text { Grind all the } \\
\text { ingredients to make a } \\
\text { past and also extract }\end{array}$ & $\begin{array}{l}\text { Paste apply on } \\
\text { the head and } \\
\text { put into nose }\end{array}$ & $\begin{array}{l}4-5 \text { drops twice } \\
\text { a day for } 7 \text { days }\end{array}$ \\
\hline & & Nari Damdari (Panchanga) & $\begin{array}{l}\text { Enicostema axillare (Poir. ex Lam.) } \\
\text { A Raynal (Gentianaceae) }\end{array}$ & $\begin{array}{l}\text { Grind and make the } \\
\text { juice mixed with deshi }\end{array}$ & Put into nose & $\begin{array}{l}\text { 4-5 drops twice a } \\
\text { day } 5 \text { days }\end{array}$ \\
\hline
\end{tabular}
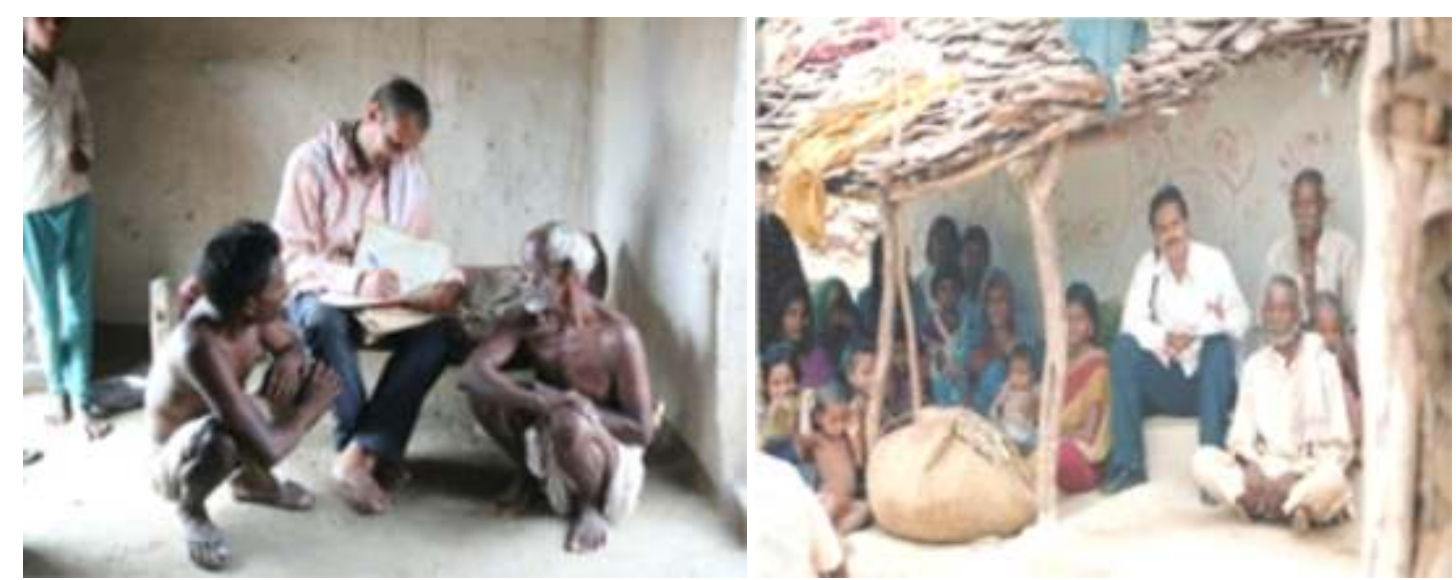

Plate. 1 Photographs of Tribal Villages of Chitrakoot during documentation

(4 formulations), Jaundice (21 formulations), Conjunctivitis (17 formulations), Mouth ulcer (15 formulations), Stomach ache (16 formulations), Ear pain (11 formulations), Skin diseases (16 formulations), Fever ( 8 formulations), Malaria fever (6 formulations), Vomiting ( 7 formulations), Loose mo- tion (15 formulations), Tooth eruption (5 formulations), Dislocation of shoulder (1 formulation), Pneumonia (9 formulations), Cough \& cold (5 formulations), Bawasir (piles) (12 formulations), Weakness (tonic) (5 formulations), Rakta Shodhan (Blood purification) (1 formulation), Safed Pradar 
(Leucorrhoea) (1 formulation), Rakta Pradar (Leucorrhoea) ( 2 formulations), Anti fertility (1 formulation), Napunsakata ( 2 formulations), Arthritis (6 formulations), Bee bite ( 1 formulation), Scorpion poison sting (4 formulations), Asthma (6 formulations), Retention of placenta (animal) (3 formulations), Cut \& wounds (4formulations), Pathari (6 formulations), Kidney failure (1 formulation), Dhat (spermatorrhoea) (2 formulations), Lakwa (Paralysis) (1 formulation), Safed dag (Leprosy) (3 formulations), Eosinophilia (1 formulation), Pimples (1 formulation), Hydrocele (1 formulation), Blindness of one eye (1 formulation), Mirgi (Epilepsy) (2 formulations), Retention of anus (1 formulation), Pechis (Dysentery) (1 formulations), Gas (Acidity) (2 formulations), Abortion (1 formulation), Rickets (1 formulations), New Born baby (1 formulations), Malnutrition (1 formulation), Headache (1 formulation), Bleeding (1 formulation), Whooping cough (2 formulations), Snake bite (1 formulation) and Adha shish (Migrane) (2 formulations). Similar study was also carried out by Singh et al. (2018) in Balaghat.

In the present study 64 plants were used as single drug and rest of 117 plants were used as compound formulations. It was also observed that different Vaidyas treats the same disease by different formulations in different villages such as malarial fever, acidity, leucorrhoea, pneumonia and paralysis etc. Out of 181 plant species, life form analysis showed that 69 species were herbs, 28 were shrubs, 53 species were trees, 22 species were climbers and 9 species were tubers. Leguminosae (Fabaceae) is the largest family contributes with 9 species, followed by Solanaceae with 6 species, Apocynaceae and Asteraceae with 5 species each, Poaceae, Malvaceae, Lamiaceae and Cucurbitaceae with 4 species each, Euphorbiaceae, Zingiberaceae and Myrtaceae with 3 species each, Piperaceae, Lythraceae, Papaveraceae, Convolvulaceae, Apiaceae, Acanthaceae, Meliaceae, Rutaceae and Menispermaceae with 2 species each and rest of 22 families having single species. It was also observed that the leaves used of 54 species, root of 39 species, tuber of 8 species, fruits of 21 species, seeds of 13 species, whole plant of 15 species, stem of 12 species, stem bark of 4 species, latex of 3 species, gum of 2 species, flower of 6 species and 4 species of oil were used for ethno-medicinal practices. The total 181 plant species belonging to 89 genera and 51 families are used in 51 different ailments and diseases.

Conclusion : The programme has drawn up and implemented for improving the traditional knowledge and economic status of the Scheduled Tribes mainly; as well as the Scheduled Caste and other backward classes were also included for the sake of their awareness. It is evident that the area is highly rich in the forest and medicinal plants as well; efforts be made to share the collected information through this project for the welfare of Scheduled Tribe of the Chitrakoot region, so that the knowledge economy could be generated, which has enough scope in this region.

\section{ACKNOWLEDGMENTS}

The authors are grateful to National Academy of Sciences, India (NASI) Prayagraj for valuable funding of the project under NASI-ST Sub plant. The cooperation of tribal people of the study site is deeply acknowledged. The authors are grateful to Shri Abhay Mahajan, Hon'ble Organizing Secretary, Deendayal Research Institute, Chitrakoot, Satna (M.P.) for providing infrastructure and necessary facilities.

\section{REFERENCES}

Chopra, R. N.; Nayar, S. L. and Chopra, L. C. (1956). Glossary of Indian Medicinal Plants. Publication and information Directorate, New Delhi.

Jain, S. K. and Rao, R. R. (1977). Dictionary of Indian folk medicine and ethnobotany: Vol. 1, Deep publication, New Delhi, p. 311.

Jain, S. K. (1991). Dictionary of Indian Folk Medicine and Ethnobotany. Deep publications, New Delhi.

Mudgal, V.; Khanna, K. K. and Hajara, P. K. (1997). Flora of Madhya Pradesh : Vol. 2. BSI Publication, Calcutta.

Sikarwar, R. L. S. (2001). Ethnobotany of Madhya Pradesh. Appl. Bot., 21(2): 133-147.

Sikarwar, R. L. S.; Pathak, Bharat and Jaiswal, Anil (2008). Some unique ethnomedicinal perceptions of tribal communities of Chitrakoot, Madhya Pradesh. Indian Journal of Traditional Knowledge, 7(4) : 613-618.

Singh, N. P.; Khanna, K. K.; Mudgal, V. and Dixit, R. D. (2001). Flora of Madhya Pradesh: Vol. 3. BSI Publication, Calcutta.

Singh, Rashmi; Koushley, P. and Khan, Suhel (2018) Participatory observation of ethno-medicinal practices by the tribes of Balaghat district of Madhya Pradesh. Life Science Bulletin, 15(2): 243-247.

Varma, D. M.; Balakrishnan, N. P. and Dixit, R. P. (1993). Flora of Madhya Pradesh: Vol. 1. BSI Publication, Calcutta. 